\title{
Complex stimulus control in humans: Merging functional and equivalence classes
}

Harold E. Lobo

West Virginia University

Follow this and additional works at: https://researchrepository.wvu.edu/etd

\section{Recommended Citation}

Lobo, Harold E., "Complex stimulus control in humans: Merging functional and equivalence classes" (2008). Graduate Theses, Dissertations, and Problem Reports. 2740.

https://researchrepository.wvu.edu/etd/2740

This Dissertation is protected by copyright and/or related rights. It has been brought to you by the The Research Repository @ WVU with permission from the rights-holder(s). You are free to use this Dissertation in any way that is permitted by the copyright and related rights legislation that applies to your use. For other uses you must obtain permission from the rights-holder(s) directly, unless additional rights are indicated by a Creative Commons license in the record and/ or on the work itself. This Dissertation has been accepted for inclusion in WVU Graduate Theses, Dissertations, and Problem Reports collection by an authorized administrator of The Research Repository @ WVU.

For more information, please contact researchrepository@mail.wvu.edu. 
COMPLEX STIMULUS CONTROL IN HUMANS:

MERGING FUNCTIONAL AND EQUIVALENCE CLASSES

\author{
Harold E. Lobo
}

Dissertation submitted to the Eberly College of Arts and Sciences

at

West Virginia University

in partial fulfillment of the requirements

for the degree of

Doctor of Philosophy

in

Psychology
Philip N. Chase, Ph.D., Chair
Barry Edelstein, Ph.D.
B. Kent Parker, Ph.D.
Michael Perone, Ph.D.
Oliver Wirth, Ph.D.

Department of Psychology

Morgantown, West Virginia
2008

Keywords: stimulus control, stimulus equivalence, functional equivalence, matching to sample, delayed prompt, college students 


\section{ABSTRACT \\ Complex Stimulus Control in Humans: \\ Merging Functional and Equivalence Classes}

Harold E. Lobo

This dissertation investigated the merging of functional and equivalence classes produced by training responding to either simple or conditional discriminations. Experiment 1 targeted the acquisition of three three-member equivalence classes featuring class-specific reinforcers and three three-member functional classes each controlling the occurrence of a nonsense vocal response. Four female college students completed testing for the targeted stimulus classes. Two subjects demonstrated classconsistent emergent responding, but only one of them maintained class-consistent responding across repeated testing. This student withdrew from the experiment before class merging was arranged. Experiment 2 simplified and expedited the procedures for producing independent emergent classes. Four female college students demonstrated class-consistent responding for three three-member equivalence classes and three two-member functional classes. Classes merged successfully after subjects completed training relating each equivalence class to a functional class via one simple or conditional discrimination. Subsequently, each merged class was expanded by training one additional simple or conditional discrimination. 


\section{Acknowledgments}

I would like to thank Phil Chase, Barry Edelstein, Kent Parker, Mike Perone, and Oliver Wirth for serving on my dissertation committee. Your suggestions contributed to improve substantially the quality of this document.

My particular gratitude to Phil Chase, who served as my advisor at WVU. His mentorship and support has proven indispensable in many more ways that I can recount. His expertise and guidance have been fundamental to most of my research endeavors. Phil is a consummate behavior

analyst and academician, but most importantly, a person of great human integrity and kindness. I am truly fortunate to have had the best advisor one could ask for!

I am thankful to Mike Perone, who taught me many valuable lessons on how to think and do research as a behavior analyst. Mike offered me lots of support as well as the invaluable opportunity to do work in his lab.

Gracias infinitas a mis padres. A ellos les debo todo lo que he logrado; sin su apoyo incondicional, sus inagotable esfuerzos y sus múltiples sacrificios nunca hubiera alcanzado esta meta. Este documento y lo que él representa no es más que un gesto de agradecimiento para con quienes siempre estaré en deuda.

I would like to thank my close friends at WVU, Chata, Mirari, Misty, and Vennessa. Your tireless support and encouragement always gave me strength to persevere.

My deep appreciation also goes to the members of the Verbal Behavior Lab. Our many lab discussions contributed greatly to my research and to my professional and academic development.

I would like to acknowledge that the Alumni Fund of the Department of Psychology supported this research. 
The surest - also the quickest - way to awake the sense of wonder in ourselves is to look intently, undeterred, at a single object. Suddenly, miraculously, it will reveal itself as something we have never seen before.

Cesare PAVESE 
Table of Contents

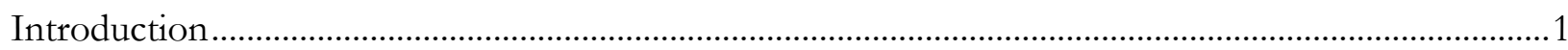

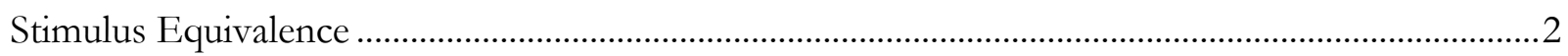

Functional Stimulus Classes and their Relation to Stimulus Equivalence..........................................

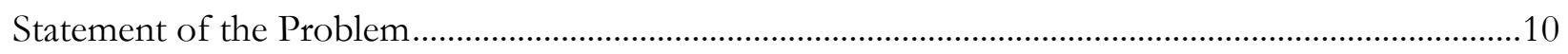

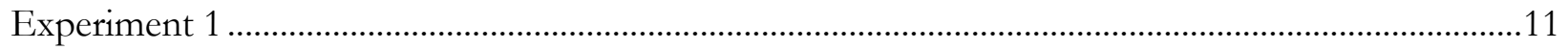

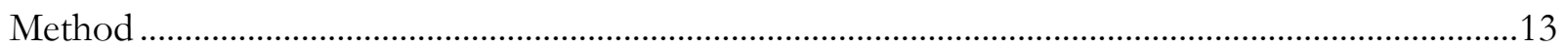

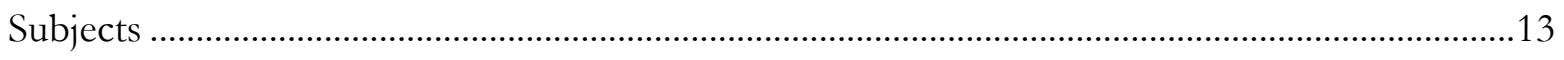

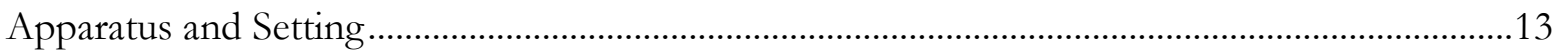

Stimuli and Target Vocal Responses .............................................................................................. 14

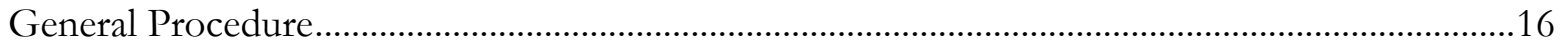

Selection of Preferred Consequences ..........................................................................................16

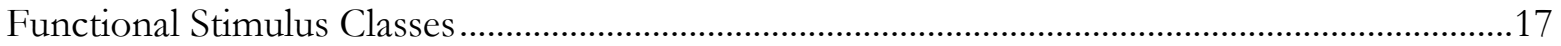

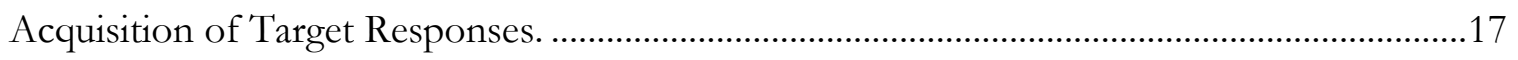

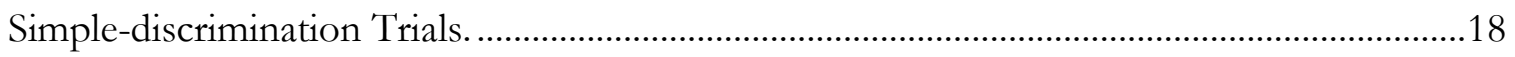

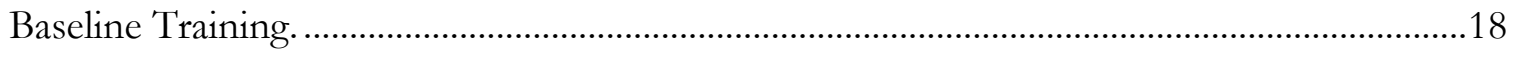

Training of New Responses required for Transfer-of-function...............................................20

Testing for Functional Equivalence. ....................................................................................20

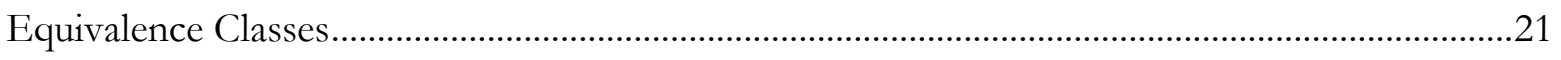

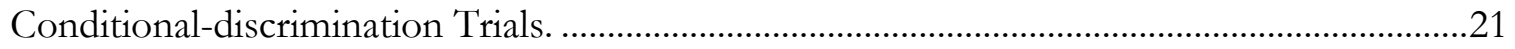

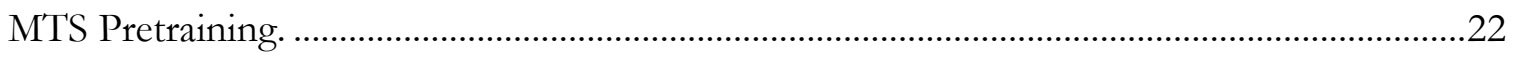

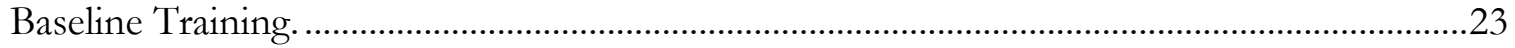

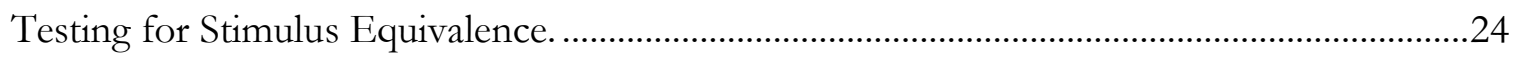

Additional Testing for Functional Equivalence........................................................................25

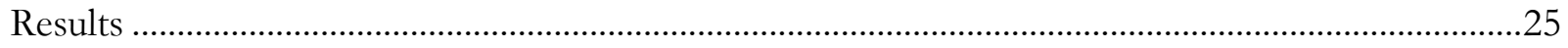

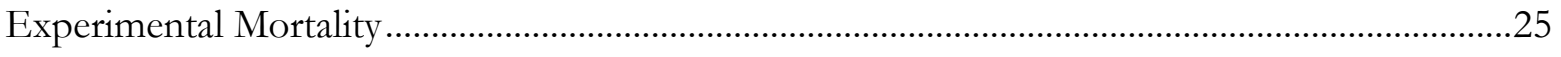

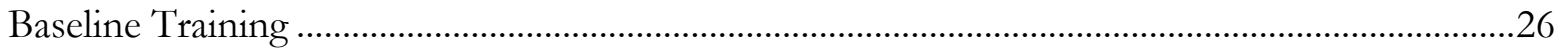

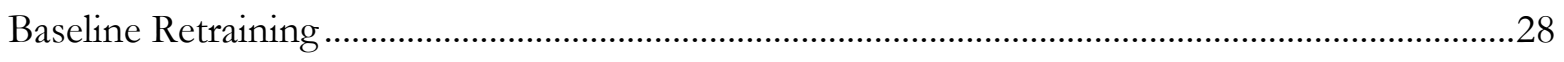

Testing for Independent Functional and Equivalence Classes ......................................................29

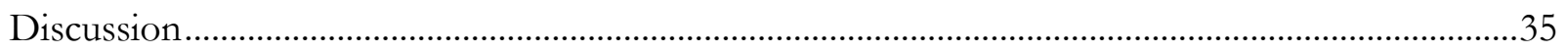




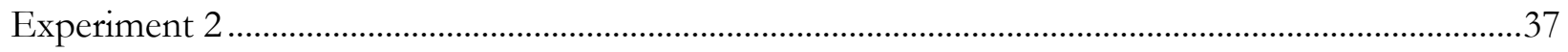

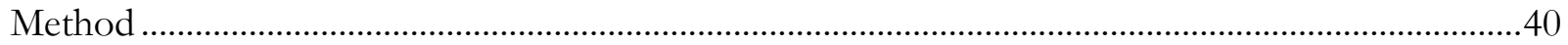

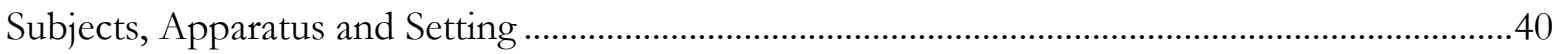

Stimuli and Target Vocal Responses ...............................................................................................40

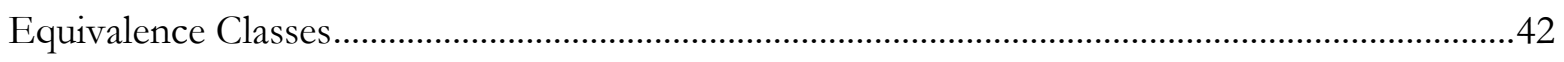

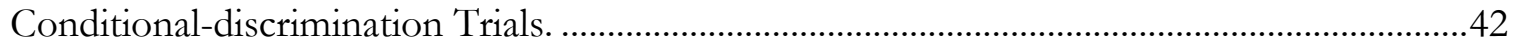

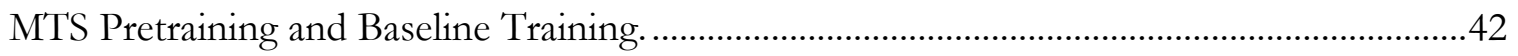

Testing for Stimulus Equivalence ………...................................................................................43

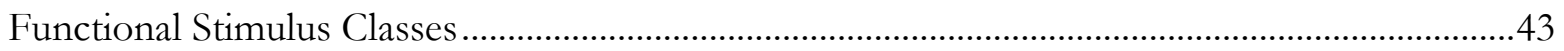

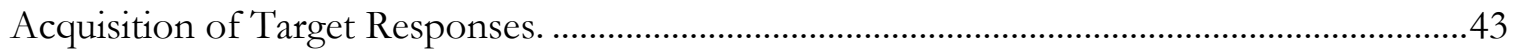

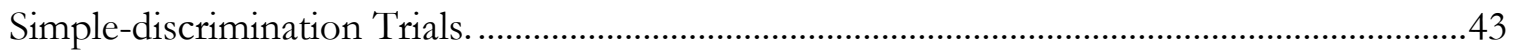

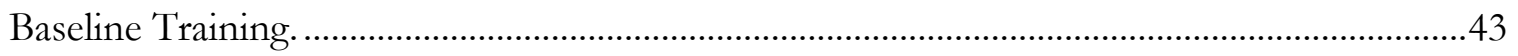

Testing for Functional Equivalence. .......................................................................................44

Additional Testing for Functional and Stimulus Equivalence .......................................................45

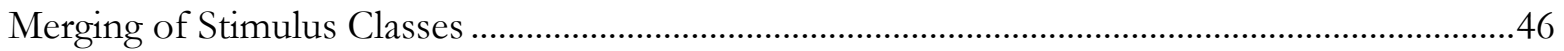

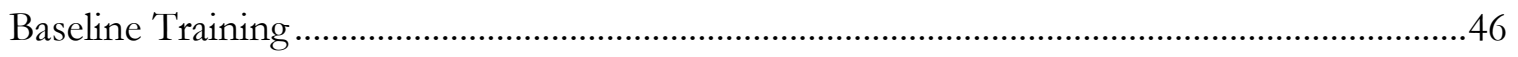

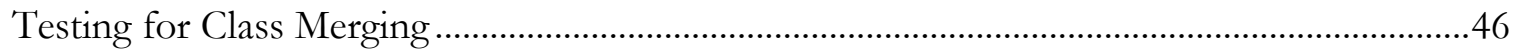

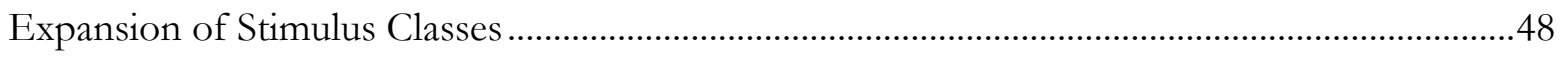

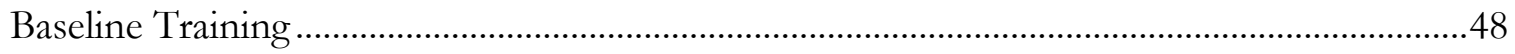

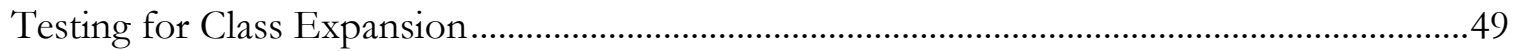

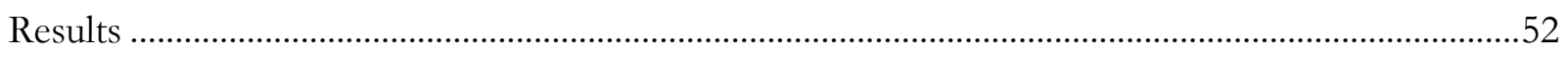

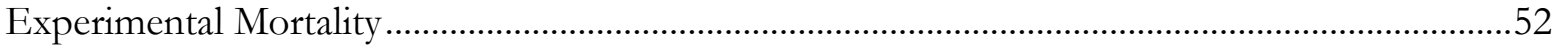

Baseline Training for Equivalence and Functional Classes .........................................................52

Baseline Retraining for Equivalence and Functional Classes...........................................................53

Testing for Independent Functional and Equivalence Classes ......................................................54

Baseline Training for Merging and Expansion of Classes ..............................................................58

Testing for Merging and Expansion of Classes ...............................................................................59

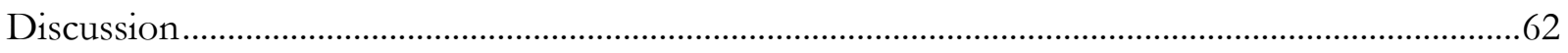

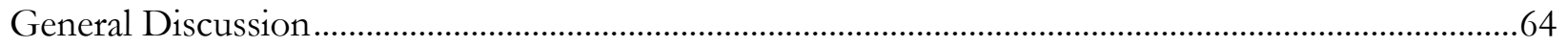

Relation between Functional and Stimulus Equivalence ................................................................64 


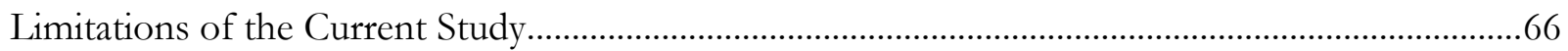

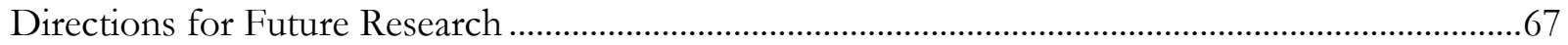

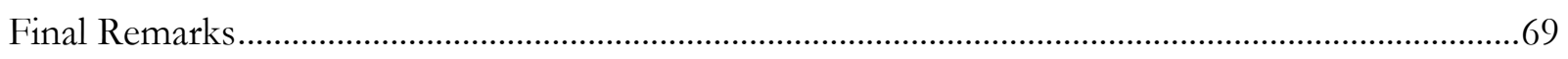

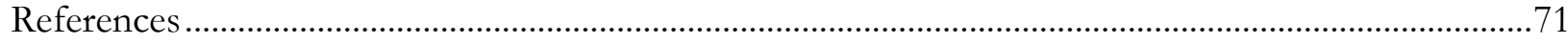

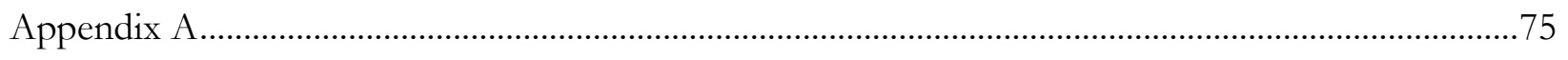

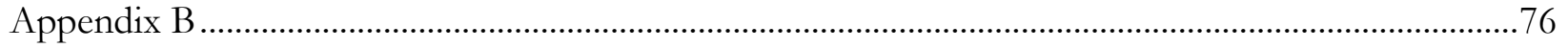

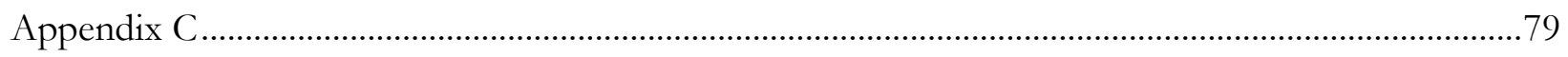

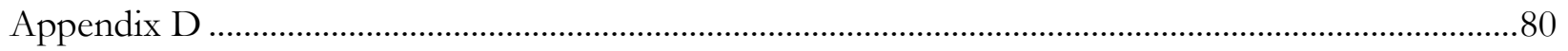

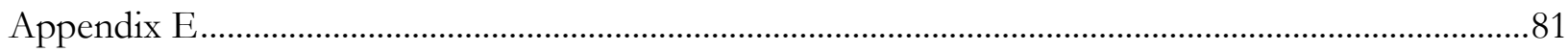

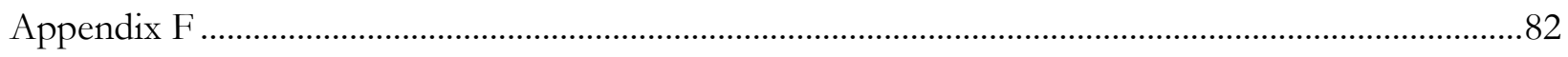

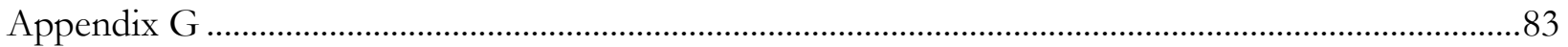




\section{List of Tables}

Table 1. Number of training trials per block and simple discriminations

trained at each stage of baseline training for functional stimulus classes in experiment 1

Table 2. Number of training trials per block and conditional discriminations

trained at each stage of baseline training for equivalence classes in experiment 1.

Table 3. Probes for emergent relations in experiment 1after baseline training

for equivalence classes...

Table 4. Blocks of trials completed by each subject at each stage of baseline

training for functional stimulus classes in experiment 1

Table 5. Blocks of trials completed by each subject at each stage of baseline

training for equivalence classes in experiment 1

Table 6. Blocks of trials completed by each subject at each stage of baseline

retraining for functional stimulus classes in experiment 1 .....

Table 7. Number of training trials per block and conditional discriminations

trained at each stage of baseline training for equivalence classes in experiment 2 .

Table 8. Baseline trials and probes for emergent relations presented during

blocks of testing for equivalence classes in experiment 2

Table 9. Number of training trials per block and simple discriminations

trained at each stage of baseline training for functional stimulus classes in experiment 2

Table 10. Baseline trials and probes for emergent relations presented

during blocks of testing for functional classes in experiment 2 .

Table 11. Baseline trials and probes for emergent relations presented to

Subjects 452 and 518 during blocks of testing for class merging in experiment 2 ........

Table 12. Baseline trials and probes for emergent relations presented to

Subjects 271 and 807 during blocks of testing for class merging in experiment 2 .

Table 13. Baseline trials and probes for emergent relations presented to

Subjects 452 and 518 during blocks of testing for class expansion in experiment 2

Table 14. Baseline trials and probes for emergent relations presented to

Subjects 271 and 807 during blocks of testing for class expansion in experiment 2

Table 15. Blocks of trials completed by each subject at each stage of baseline training for equivalence classes in experiment 2 ....... 
Table 16. Blocks of trials completed by each subject at each stage of baseline training for functional stimulus classes in experiment 2 ...

Table 17. Blocks of trials completed by Subjects 452, 518 and 807 at each stage of baseline retraining for functional stimulus classes in experiment 2

Table 18. Blocks of trials completed by each subject at each stage of baseline training for merging and expansion of classes in experiment 2 . 


\section{List of Figures}

Figure 1. All possible ordered pairs that illustrate equivalence relations from simple and conditional discriminations when class specific reinforcers are used

Figure 2. Schematic diagram of the general design for Experiment 1

Figure 3. Stimuli and target responses used in Experiment 1

Figure 4. Business logos available for the subjects to select as preferred consequences in Experiment 1

Figure 5. Mean percent correct responding for baseline and probe trials

during initial testing for functional equivalence for Subject 412

Figure 6. Mean percent correct responding for baseline and probe trials

during testing for stimulus equivalence for Subject 412

Figure 7. Mean percent correct responding for baseline and probe trials

during additional testing for functional equivalence for Subject 412 .......

Figure 8. Mean percent correct responding for baseline and probe trials

during testing for functional equivalence for Subject 718 .

Figure 9. Mean percent correct responding for baseline and probe trials

during testing for stimulus equivalence for Subject 851

Figure 10. Mean percent correct responding for baseline and probe trials

during initial testing for functional equivalence for Subject 315

Figure 11. Mean percent correct responding for baseline and probe trials

during testing for stimulus equivalence for Subject 315

Figure 12. Mean percent correct responding for baseline and probe trials during additional testing for functional equivalence for Subject 315 ......

Figure 13. Schematic diagram of the general design for Experiment 2.

Figure 14. Stimuli and target responses used in Experiment 2.

Figure 15. Mean percent correct responding for baseline and probe trials during testing for stimulus equivalence for Subject 452

Figure 16. Mean percent correct responding for baseline and probe trials

during testing for functional equivalence for Subject 452

Figure 17. Mean percent correct responding for baseline and probe trials during testing for stimulus equivalence for Subject 518 
Figure 18. Mean percent correct responding for baseline and probe trials during testing for functional equivalence for Subject 518 .56

Figure 19. Mean percent correct responding for baseline and probe trials during testing for stimulus equivalence for Subject 271

Figure 20. Mean percent correct responding for baseline and probe trials during testing for functional equivalence for Subject 271

Figure 21. Mean percent correct responding for baseline and probe trials during testing for stimulus equivalence for Subject 807

Figure 22. Mean percent correct responding for baseline and probe trials during testing for functional equivalence for Subject 807

Figure 23. Mean percent correct responding for baseline and probe trials during testing for merging and expansion of classes for Subject 452.

Figure 24. Mean percent correct responding for baseline and probe trials during testing for merging and expansion of classes for Subject 518.

Figure 25. Mean percent correct responding for baseline and probe trials during testing for merging and expansion of classes for Subject 271

Figure 26. Mean percent correct responding for baseline and probe trials during testing for merging and expansion of classes for Subject 807. 


\section{Introduction}

A stimulus class is a set of stimuli for which a response occurs in the presence of all stimuli in the class and differentiated responding occurs to stimuli outside the class (Keller \& Schoenfeld, 1950). Although direct reinforcement of responding to a set of stimuli can produce class membership, numerous studies also have been conducted on emergent stimulus classes. In these classes, responding specific to relations among stimuli is obtained in the absence of a history of explicit reinforcement. Emergent classes often are distinguished based on whether class members share formal characteristics or not. If behavior is controlled by novel stimuli via primary generalization, then the stimuli constitute a perceptual class. These classes are characterized by the physical similarity shared among members of the class. In contrast, if behavior is controlled by novel stimuli related by direct contingencies of reinforcement, the stimuli are considered members of an arbitrary stimulus class.

Studies on emergent arbitrary classes are relevant for understanding adaptive behavior in the presence of novel environmental events (Chase, 2003; Shahan \& Chase, 2002). Arbitrary stimulus classes also provide a model for investigating generative aspects of language (cf., Chomsky, 1959), and research on relations between stimulus equivalence and functional stimulus classes (both defined below) can contribute to a behavior analytic account of complex human behavior (see Hayes, BarnesHolmes, \& Roche, 2001; Pilgrim \& Galizio, 1996). For instance, stimulus equivalence has been considered as a model for symbolic representation and semantic correspondence (Sidman, 1986, 1994), and functional classes have been considered akin to syntactic relations (Lazar, 1977).

Although conceptual and procedural differences between emergent functional and equivalence classes usually are emphasized, these differences should not obscure their fundamental similarities (Dougher \& Markham, 1994, 1996). To further our understanding of these similarities, this dissertation investigated the merging of equivalence and functional stimulus classes. In order to 
justify this investigation, definitions of functional and equivalence classes are reviewed along with studies relating both types of classes.

\section{Stimulus Equivalence}

Equivalence classes usually are examined through matching-to-sample (MTS) procedures. MTS trials typically begin by presenting a sample stimulus (e.g., A1). Once an observing response to the sample occurs (e.g., pressing A1), a set of comparison stimuli (e.g., B1, B2, and B3; i.e., the set of B stimuli) appear. The individual then selects one of the comparisons. When differential consequences are provided, selection of the comparison designated as correct by the experimenter (hereafter referred to as correct comparison) is followed by delivery of a reinforcer. MTS trials entail conditional discriminations. In a conditional discrimination, a specific sample (conditional stimulus) sets a given comparison as a discriminative stimulus for responding that produces reinforcement (e.g., selecting $\mathrm{B} 1$ from the set of $\mathrm{B}$ stimuli in the presence of $\mathrm{A} 1$, also referred to as the A1B1 conditional discrimination). For stimulus equivalence to be tested, two or more baseline conditional discriminations (e.g., A1B1 and B1C1) must be trained directly among stimuli that share no obvious physical similarity to one another.

An equivalence class is demonstrated when reflexive, symmetric, and transitive stimulus relations emerge during tests conducted in the absence of direct reinforcement (Sidman et al., 1982; Sidman \& Tailby, 1982). Reflexivity requires each member of the class to be matched to itself (i.e., generalized identity MTS; e.g., A1 is matched to A1, B1 to B1, and C1 to C1). Symmetry describes a bidirectional conditional relation. Here, stimuli presented as samples during baseline training now serve as correct comparisons and previous comparisons serve as samples. For instance, if B1 was matched to A1 and C1 was matched to B1 during baseline training, symmetry is demonstrated by matching A1 to B1 and B1 to C1. Transitivity encompasses demonstrating an emergent relation between the sample of one baseline conditional discrimination and the correct comparison of 
another baseline conditional discrimination (e.g., C1 is matched to A1). Additionally, symmetry and transitivity can be tested together in probes for combined symmetry and transitivity (hereafter referred to as a combined relation). For example, given training on baseline conditional discriminations $\mathrm{A} 1 \mathrm{~B} 1$ and $\mathrm{B} 1 \mathrm{C} 1$, the respective test for a combined relation will require $\mathrm{A} 1$ to be matched to C1.

Equivalence classes can be expanded through MTS procedures. In class expansion, additional stimuli become interchangeable with members of an equivalence class by directly relating the new stimuli with at least one member of each targeted class (Green \& R. R. Saunders, 1998). In one study on class expansion, R. R. Saunders, Drake, and Spradlin (1999, Exp. 2) established two five-member equivalence classes with one individual after training of BA, CA, DA, and EA sets of conditional discriminations. Subsequently, R. R. Saunders et al. introduced two novel stimuli (F1 and F2) by training the FA set of conditional discriminations. Subject's responding during probes for emergent relations between the stimuli F1 and F2 and the remaining sets of stimuli demonstrated the expansion of the equivalence classes.

Classes can be expanded also by using class-specific reinforcers. In these studies, a different reinforcer is used for each class during training of baseline conditional discriminations and when introducing new stimuli to the classes (for a review, see McIlvane, Dube, Kledaras, de Rose, \& Stoddard, 1992). For example, Dube, McIlvane, Mackay, and Stoddard (1987, Exps. 2 \& 3) provided baseline training with class-specific reinforcers (different food items) to two individuals. Subjects demonstrated two three-member equivalence classes involving auditory and visual stimuli. Subjects then received identity MTS training with one novel stimulus per class using class-specific reinforcers. High accuracy was observed on class-expansion probes that featured the novel stimuli. Classconsistent responding also occurred on probes in which stimuli presented as reinforcers served as samples or comparisons, suggesting that these stimuli became class members as well. 
Additionally, equivalence classes can be merged. Merging is different from expansion in that, instead of adding class members to existing classes, two or more classes are combined. Thus, merging is defined by the union of two classes featuring non-overlapping stimuli. In one study on merging, Sidman, Kirk, and Willson-Morris (1985, Exp. 2) established six three-member equivalence classes with two individuals. Half of the classes featured one set of stimuli; whereas the remaining classes involved a second stimulus set. Then, subjects completed additional training with three new conditional discriminations, each relating a sample from one stimulus set with a correct comparison from the second set. Probes for emerging relations showed that previously established equivalence classes merged across the stimulus sets, leading to three six-member equivalence classes.

Although this study demonstrated that equivalence classes can be merged, it did not include any systematic manipulation of the variety of stimulus relations that may lead to class union. For instance, Sidman et al. (1985) did not assess the effectiveness of different sets of conditional discriminations on producing class merging. Additionally, established classes were merged simultaneously, which prevented successive within-subject replications of this manipulation. As an alternative, a third class or novel stimuli could have been added to each merged class. In this regard, further exploration of the kind of stimulus relations that may lead to class union is warranted. Theoretical observations in this regard will be presented once relations between equivalence and emergent functional stimulus classes are examined.

\section{Functional Stimulus Classes and their Relation to Stimulus Equivalence}

Stimuli are considered part of a functional class when they control the occurrence of the same behavior or operant (Goldiamond, 1962, 1966). Members of an emergent functional stimulus class are interchangeable in that "contingencies applied to one member of the class will affect other members of the class [without explicit training]" (Goldiamond, 1962, p. 303). These classes may involve stimuli that share no perceptual similarity and therefore constitute arbitrary stimulus classes. 
Although substitutability of functional class members for one another might suggest a connection with stimulus equivalence, procedural differences between both kinds of classes have been emphasized (cf., Wirth \& Chase, 2002). For instance, functional classes are examined typically through simple discriminations. In a simple discrimination, the occurrence of a specific response in the presence of a stimulus $(\mathrm{S}+)$ will be reinforced; whereas, in the presence of a different stimulus $(\mathrm{S}-)$, the response will not produce the reinforcer. Given a set of stimuli that serve as $\mathrm{S}+$ for a particular response, an emergent functional stimulus class would be demonstrated if, after training one $\mathrm{S}+$ for a new response, the remaining $\mathrm{S}+$ stimuli control the occurrence of the new response in the absence of direct conditioning while other stimuli serve as S-. This test for class formation is known as transfer-of-function, also called transformation of function, which refers to the emergent acquisition of a stimulus function by members of a stimulus class, provided that the function has been conditioned explicitly to at least one class member (Dougher \& Markham, 1994, 1996). Given that the current study is concerned primarily with emergent stimulus classes, hereafter, all references to functional classes will allude to emergent stimulus classes consistent with the procedures introduced above.

In one study on functional stimulus classes, Vaughan (1988) presented pigeons with a set of 40 stimuli in which one half of the stimuli served as $\mathrm{S}+$ for food reinforcement and the remaining half served as S-. Once subjects reliably discriminate between the two groups of stimuli, Vaughan reversed the contingencies for each group. Successive reversals continued, each after consistent discrimination of both groups of stimuli was attained. After repeated contingency reversals, the number of errors following the changed contingencies decreased gradually. Eventually, the pigeons shifted their responding quickly according to the contingencies for the two groups of stimuli. Functional stimulus classes were demonstrated insofar as contact of one class member with the reversed contingencies was sufficient for the remaining class members to control responding 
according to the new contingencies. Following a model of equivalence based on set theory, Vaughan argued that the subjects' partitioning of the stimuli into two subsets implied an equivalence relation among the members of each subset (see Hrbacek \& Jech, 1999). As Sidman (1994) pointed out, turning a set of elements into partitions requires a basis for that separation, and "with respect to that basis, the elements of the group are equivalent to each other" (p. 417, italics are from the original).

Sidman, Wynne, Maguire, and Barnes (1989) tested Vaughan's (1988) suggestion that functional classes where composed of equivalent stimuli by explicitly testing members of a functional class using MTS and tests for equivalence-class formation. For three adults, Sidman et al. used Vaughan's contingency reversal procedure to establish two functional stimulus classes of three stimuli each (class A1, B1, and C1; and class A2, B2, and C2). Sidman et al. then provided these individuals with identity MTS training for two stimuli (A1, C1; and A2, C2) from each functional class. Afterwards, the authors conducted probes that demonstrated the emergent conditional discriminations A1C1, C1A1 and A2C2, C2A2. Then, Sidman et al. added two new stimuli (D1, E1 and D2, E2) to the each class by training the conditional discriminations D1A1, E1D1 and D2A2, E2D2. For two of the subjects, combined symmetry and transitivity was demonstrated with probes for the emerging conditional discriminations B1D1, C1E1 and B2D2, C2E2. Thus, Sidman (1994) concluded that "functional classes do imply equivalence relations in behavior, in spite of different definitions and testing procedures" (p. 421, italics are from the original).

Chase, Ellenwood, and Madden (2008) provided an initial demonstration of merging of functional and equivalence classes in an attempt to develop an analogue of naturally occurring semantic and syntactic relations. In this study, three individuals initially completed MTS training with 24 Spanish words. This training produced 12 stimulus pairs. A three-position sequence training procedure (Lazar, 1977) followed for six of the stimuli that were not paired during the previous training. In this procedure, stimuli differed in their function in the sequencing task, with two stimuli 
serving in each sequence position. That is, given three stimuli presented from left to right in random order, the stimulus that served in the first position was selected first, followed by the stimulus for the second position, and finishing with the stimulus serving in the third position. This sequence was analogous to responding to a simple syntactic relation between nouns, adjectives, and verbs in a declarative sentence. Chase et al. then related one stimulus per position to two other stimuli through MTS trials. Three equivalence classes of eight stimuli each were subsequently demonstrated.

Functional classes also were demonstrated when members of each equivalence class shared the same function (i.e., position in sequencing trials) during tests for novel verbal sequences. Chase et al. conceptualized the equivalence classes as illustrating emergent semantic relations and the functional classes as exemplifying emergent syntactic relations.

As an investigation of merging of functional and equivalence classes, the study by Chase et al. (2008) has limitations that call for further research. First, given that no independent variable was manipulated, this study was a demonstration of transfer of specific functions across stimuli related through MTS training rather than a formal experiment on class union. Additionally, probe performance was equivocal for one subject. Chase et al. also used Spanish words as stimuli, which left uncontrolled potential pre-experimental historical effects. Most importantly, the procedures followed by Chase et al. did not allow the experimental isolation of environmental factors responsible for merging of equivalence and functional classes.

These observations on research by Chase et al. (2008), then, are similar to those drawn earlier about the merging of equivalence classes: studies on class union require the systematic manipulation of the variables that are purported to produce this effect. In this regard, Sidman (2000) proposed that equivalence classes, as well as the expansion and merging of classes, are a direct outcome of the contingencies of reinforcement. These contingencies, according to Sidman, relate all stimuli and responses that lead to reinforcement and, therefore, equivalence relations should be 
found among all ordered pairs of elements that are correlated with a reinforcer in these contingencies (e.g., Dube et al., 1987).

This formulation comes from set theory, in which relations between objects of two kinds are defined using ordered pairs (Halmos, 1960; Hrbacek \& Jech, 1999). An ordered pair refers to a sequence of two different elements (e.g., the pair $(a, b))$ in which one and only one element is the first coordinate of the pair (e.g., a), and one and only one element is the second coordinate (e.g., b). As noted above, set theory defines a partition as an equivalence class if all elements in the partition conform to the relations of reflexivity, symmetry, and transitivity. Consequently, on the basis that all elements of the partition share these relations, an equivalence class is described exhaustively if all possible ordered pairs among its members are listed. Each of these ordered pairs would correspond to a unique sequence of two equivalent elements (Halmos; Hrbacek \& Jech).

Figure 1 illustrates the relations among ordered pairs entailed by the simple discrimination procedures used to generate functional classes and by the conditional discrimination procedures used to produce stimulus equivalence. In Figure 1, stimuli and responses correlated with reinforcer delivery (i.e., the positive elements of each contingency of reinforcement) are presented in bold face. These simple and conditional discriminations show four separate classes. Each class features a specific reinforcer and is denoted by a different numeral (e.g., $S^{\mathrm{R}} 1, \mathrm{~S}^{\mathrm{R}} 2$ ). Notice how the classes include all possible combinations of pairs among stimuli and responses correlated with delivery of a reinforcer. For example, the ordered pairs $A 1 R 1, R 1 S^{R} 1$, and $A 1 S^{R} 1$ belong to the same class, but this class does not include ordered pairs such as $S^{\mathrm{R}} 2 \mathrm{~A} 2, \mathrm{~B} 3 \mathrm{~A} 3$, or $\mathrm{A} 4 \mathrm{~S}^{\mathrm{R}} 4$. 
Simple Discriminations

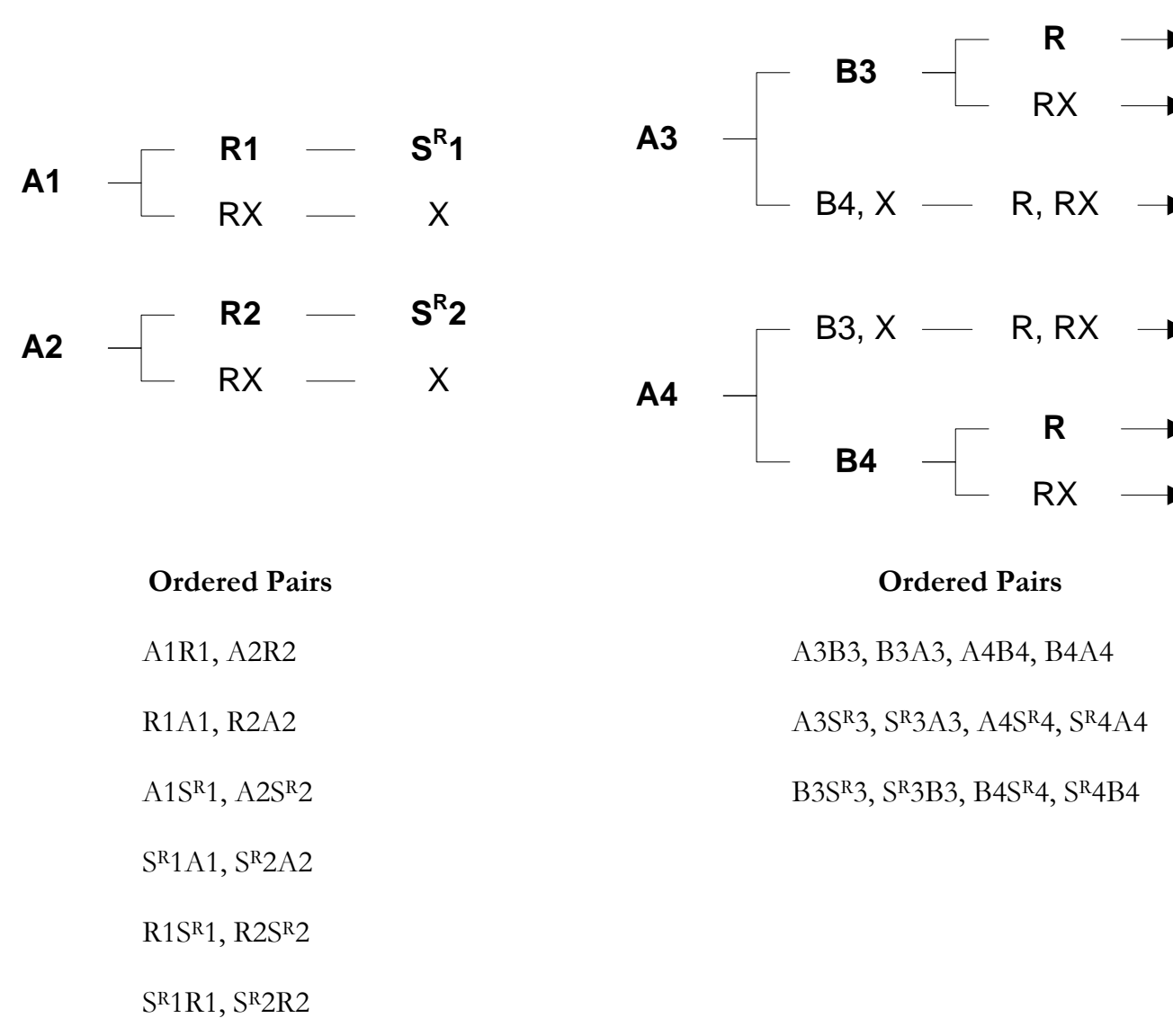

Figure 1. All possible ordered pairs that illustrate equivalence relations from simple and conditional discriminations when class specific reinforcers are used. Bold symbols indicate positive elements of each contingency, " $\mathrm{S}$ " refers to a reinforcer with numbers denoting its respective class, " $\mathrm{X}$ " denotes any undetermined stimulus or response.

According to Sidman (2000) class expansion should occur after training a relation between a novel stimulus and any of the stimuli in any of the ordered pairs. For example, by training a novel conditional discrimination between the stimulus in the left column, A1, and a new stimulus B1, class expansion will be demonstrated if probes show the emergence of additional relations, for example $\mathrm{B} 1 \mathrm{R} 1$ and $\mathrm{S}^{\mathrm{R}} 1 \mathrm{~B} 1$, but not B1R2 and $\mathrm{S}^{\mathrm{R}} 2 \mathrm{~B} 1$. Additionally, merging should occur as a function of establishing at least one ordered pair of elements that relates one class to the other. For example, class 1 can be merged with class 3 by training a simple discrimination featuring the ordered pair 
A3R1 or B3R1, or by training a conditional discrimination presenting the ordered pair A1A3 or $\mathrm{B}_{3} \mathrm{~S}^{\mathrm{R}} 1$. Thus, ordered pairs might be manipulated to determine the conditions responsible for class union. Previous research has been limited to exploring only one of many possible ordered pairs leading to class union, that is, previous studies have focused exclusively on training conditional discriminations involving antecedent stimuli from separate classes. It remains to be established whether manipulating other ordered pairs will result also in merging of classes.

\section{Statement of the Problem}

Examining how arbitrary stimulus classes are formed is important for understanding generative behavior. Although the studies by Sidman et al. (1985, Exp. 2) and by Chase et al. (2008) illustrated the complex symbolic relations that may emerge from the union of different types of arbitrary stimulus classes, procedural weaknesses prohibit a more thorough understanding of the factors responsible for this effect. This dissertation, therefore, investigated whether independent functional and equivalence classes merged under conditions in which pre-experimental historical variables were controlled and purported features of the environment that facilitate merging were manipulated experimentally.

Following Sidman's (2000) descriptions of how equivalence relations are established, this dissertation examined whether class union is the product of training responding any ordered pairs created between a member of one class and a member of another. Of particular interest is the union of emergent arbitrary classes produced by training ordered pairs involving either simple or conditional discriminations. Specifically, this dissertation focused on whether: a) a class-specific consequence from an equivalence class could be trained as a discriminative stimulus for a response controlled by a functional class, b) an antecedent stimulus from an equivalence class could be trained as discriminative stimulus for a response controlled by a functional class, and c) a conditional discrimination could be trained between a stimulus from an equivalence class and a stimulus from a 
functional class. In line with Sidman's analysis on the formation of equivalence relations, training of any of these discriminations should be sufficient to produce transfer-of-function across the functional and the equivalence classes. Two experiments based on the procedures by Wirth and Chase (2002) were conducted to evaluate these possibilities.

\section{Experiment 1}

The purpose of Experiment 1 was to investigate one type of ordered pair that is predicted to produce merging of arbitrary classes. Specifically, given independent functional and equivalence classes, training of a simple discrimination between a stimulus used as class-specific reinforcer during baseline training for each equivalence class and a response from a functional class should lead to the union of these classes.

The general design for this experiment is presented in Figure 2. Experiment 1 began by teaching subjects a set of vocal responses consisting of nonsense consonant-vowel-consonant (CVC) syllables. These responses were used for training simple discriminations leading to testing of functional stimulus classes through transfer-of-function tests. Subjects also completed training and testing for equivalence classes featuring a different set of stimuli. As described below, problems with subjects' attendance and performance began to occur at the seventh and eighth steps listed in Figure 2, which corresponded to testing for equivalence classes and for functional classes before linking these emergent classes. Subjects withdrew themselves from the experiment or failed to maintain class-consistent responding for the independent functional and equivalence classes (details are presented in the Results section). As a result, all subsequent steps of this experiment were not conducted. What follows is a detailed description of the methods used up to the tests for class merging (the ninth step in Figure 2). 


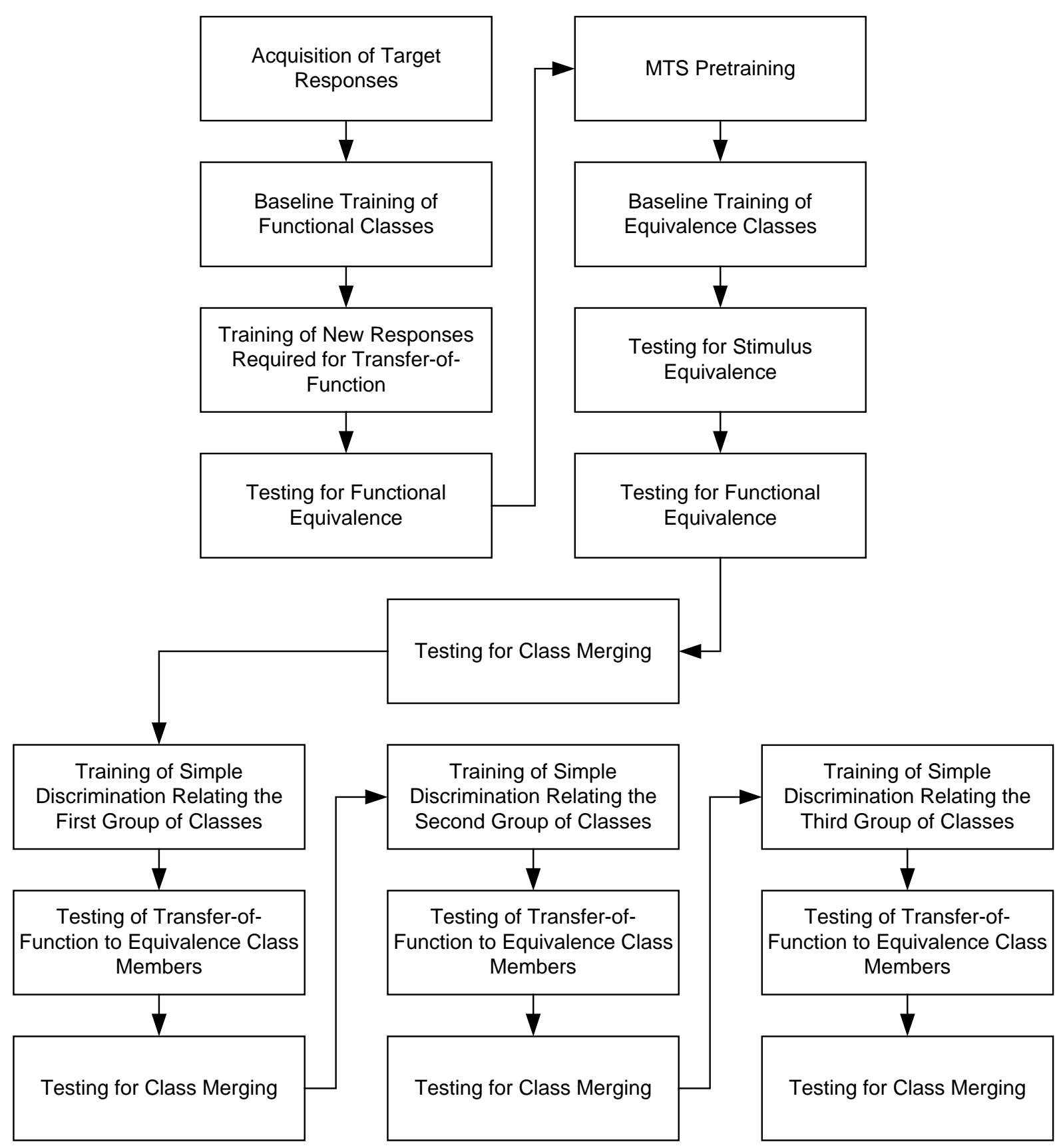

Figure 2. Schematic diagram of the general design for Experiment 1. 


\section{Method}

Subjects

Nine female West Virginia University (WVU) undergraduate students participated in this study. Subjects were selected randomly from students recruited at WVU's Department of Psychology (Appendix A shows the recruitment form). Subjects lacked prior history with similar experiments. Subjects read and signed a Consent and Information Form (Appendix B) prior to engaging in any experimental activities. Subjects were compensated based on their performance and attendance during the study. Compensation based on performance depended on the number of correct responses (defined below) emitted by the subject. Each response was worth $\$ 0.01$. Additionally, $\$ 3.00$ bonus was added to the subject's earnings for every session attended. Subjects also received extra-credit slips valid in their Psychology course(s). Attendance earnings and extracredit slips were provided to students who were dismissed by the experimenter. Subjects were eligible for their performance earnings even if they withdraw from the experiment. Monetary earnings were planned to be delivered as gift certificates, although subjects opted to receive their earnings in the form of a personal check. Students received all awarded forms of compensation after their participation in the study ended.

\section{Apparatus and Setting}

The experiment was carried out by an application programmed in Microsoft ${ }^{\circledR}$ Visual Basic ${ }^{\circledR}$ 6.0. This application interfaced with Microsoft ${ }^{\circledR}$ Speech SDK 5.1. A Pentium ${ }^{\circledR}$-class computer ran the program. A 35.5-cm color monitor with a screen resolution of 1024 by 768 pixels displayed the stimuli. A two-button wheel mouse and a microphone served as input devices. The left button controlled all actions for the mouse. Sessions were conducted separately for each subject in a soundattenuated room measuring approximately $180-\mathrm{cm}$ by $180-\mathrm{cm}$. The room contained a large desk, a chair, a computer, a mouse, and a headset featuring a noise-cancelling microphone (.Audio ${ }^{\text {TM }} 350$, 
Plantronics ${ }^{\circledR}$, Santa Cruz, CA). The room included a $30-\mathrm{cm}$ by $30-\mathrm{cm}$ wooden door located to the right of the desk that was opened when the experimenter communicated with the participants. A 117-cm by 50.8-cm one-way mirror, situated to the right of this door, allowed the experimenter to observe the behavior of the subjects from a control room. A two-port video splitter (GVS92, Iogear ${ }^{\circledR}$, Irvine, $\left.C A\right)$ arranged for the video signal from the subject's monitor to be displayed on a dedicated monitor located in the control room. White noise was played during experimental sessions to mask extraneous noises.

\section{Stimuli and Target Vocal Responses}

Stimuli consisted of 21 figures. Eighteen of these stimuli corresponded to abstract figures divided into two stimulus sets. Stimulus set 1 was used for establishing functional stimulus classes; whereas stimulus set 2 was used for equivalence classes. A notation featuring a capital letter and a number (e.g., A1) identified each stimulus. Stimuli A through F, presented in Figure 3, designated sets of comparison stimuli or of simultaneously trained stimuli that served as antecedent stimuli during training of both types of classes (e.g., B1, B2, and B3 corresponded to set of B stimuli). The numbers used to designate stimuli corresponded to the stimulus classes that may be established after merging the functional and equivalence classes (e.g., A1, B1, C1, D1, E1, F1, and G1 would be members of the 1's class.). The three G stimuli, G1, G2, and G3, served as class-specific reinforcers for the baseline trials of the equivalence classes. These stimuli consisted of business logos selected by the subject from the options shown in Figure 4. Figure 3 also shows the six responses targeted in this study. Responses consisted of nonsense CVC syllables. Specifically, responses "Mek" and "Gox" were assigned to class 1, "Ruq" and "Nal" to class 2, and "Tib" and "Vak" to class 3. Subjects did not have access to this nomenclature. 


\section{Stimulus Set 1}

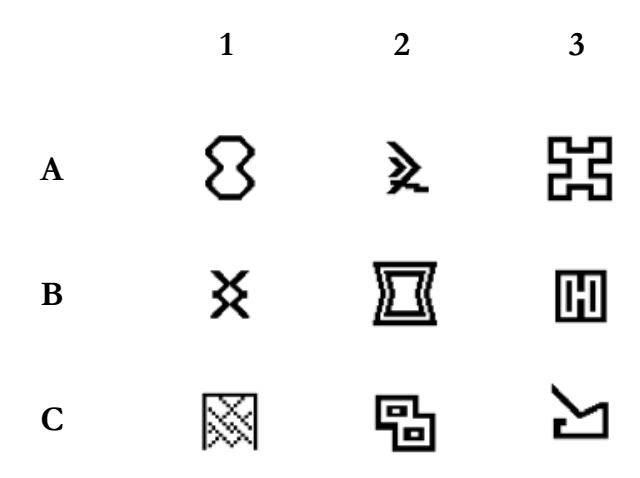

Stimulus Set 2

\begin{tabular}{|c|c|c|}
\hline & 1 & 2 \\
\hline D & 勉 & $\tilde{n}$ \\
\hline $\mathbf{E}$ & 句 & 黾 \\
\hline$F$ & $\Delta$ & 凅 \\
\hline
\end{tabular}

\section{Target Responses}

$1 \quad 2 \quad 3$

$\begin{array}{llll}\text { Rsp 1 } & \text { Mek } & \text { Ruq } & \text { Tib } \\ \text { Rsp 2 } & \text { Gox } & \text { Nal } & \text { Vak }\end{array}$

Figure 3. Stimuli and target responses used in Experiment 1. Sets of stimuli A through F correspond to the stimuli used during training of functional and equivalence classes for Experiment 1. The target responses refer to class-specific responses used in Experiment 1. 


\section{General Procedure}

Sessions lasted approximately 1 hour and 15 minutes. Sessions consisted of a variable number of blocks of trials. As many blocks as possible were completed during a session. Blocks of trials consisted of a number of simple discrimination and/or conditional-discrimination trials conducted according to the procedures described below. Prior to a session, subjects were asked to leave their watches and other personal effects with the experimenter. Subjects also were asked to turn off their mobile phones and any other portable electronic devices they carried. During each session, subjects were provided with the option of taking a 5 minute break after approximately half of the session had elapsed. At the beginning of each block of trials, the computer presented a welcome message (Appendix C).

\section{Selection of Preferred Consequences}

At the onset of the experiment, each subject was asked to choose four preferred logos corresponding to the businesses for which the subject would earn gift certificates. Initially, the welcome message appeared on the computer screen. Subsequently, the subject was instructed by the computer to select four preferred business logos from among eight alternatives. These instructions stated that the selected logos would be used for rewarding the subject's performance and participation in the study (see Appendix D). Logos were displayed on the computer screen along a grid composed of two rows of four elements each. On top of the grid, an instruction stating: "Please select four preferred businesses" was shown. Each logo was presented on a white-background button. Upon selection of a logo, its background darkened and the button became inoperative. After four logos were selected, a new screen displayed the chosen logos and the subject was allowed to verify her selections by answering the question: "Are these the businesses you want to select?" This question was displayed on top of the logos along with buttons for affirmative and negative answers. Once the subject made her final selections, one of the selected logos was randomly chosen to serve 
as a consequence for the functional classes and for MTS pretraining. The remaining logos were randomly assigned to serve as consequences for the equivalence classes and as the set of $\mathrm{G}$ stimuli during the rest of the experiment.

\section{BARNES\&NOBLE AÉrOPOSTALE

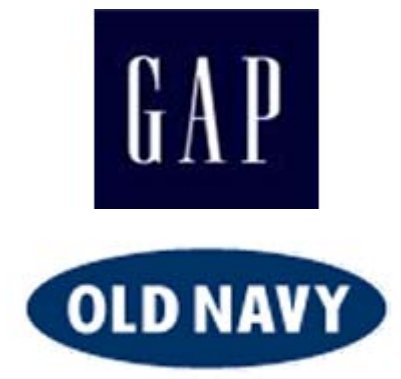 \\ Bath \&BodyWorks
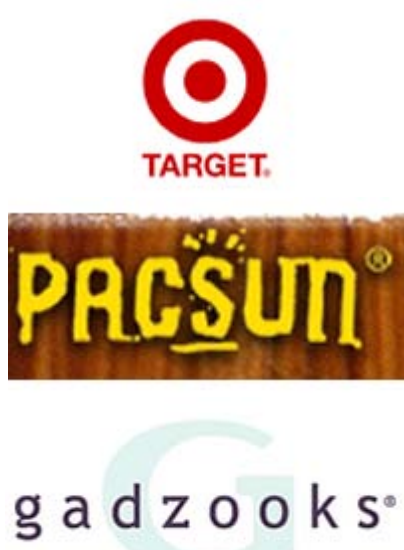

Figure 4. Business logos available for the subjects to select as preferred consequences in Experiment 1. Business logos corresponded to gift certificates made available to the subjects based on their performance and attendance in Experiments 1 and 2.

\section{Functional Stimulus Classes}

Acquisition of target responses. Prior to training the functional classes, a block of trials was completed to accurately identify each subject's utterances of all target responses. A procedure similar to that described by Wirth, Chase, \& Munson (2000) was followed. First, a sample of each target response was recorded by prompting the subject to say each of the responses aloud five times in succession (e.g., "Say 'Gox"). Subsequently, the subject was prompted to say each response one at a time in random order. Vocal responses corresponding to the response targeted in a given trial (hereafter referred to as correct responses) produced a distinctive 0.75-s tone. Simultaneously, a business logo appeared for $1.5 \mathrm{~s}$ on the computer screen and one cent was added to earnings 
towards the gift certificate corresponding to the displayed logo. Instances of target responses other than the correct response (hereafter referred to as incorrect responses) were followed by a different 0.75-s tone and simultaneous presentation of a red $\mathrm{x}(\mathbf{x})$ for $1.5 \mathrm{~s}$. Utterances that do not correspond reliably to any target response were considered unrecognizable. Following unrecognized utterances, a subject was prompted to say the target response by presenting this instruction for $1.5 \mathrm{~s}$ : "Not recognized, please try again." Subsequently, the prompt that produced the unrecognized utterance appeared again. If a vocal response did not register within $20 \mathrm{~s}$, the subject was prompted to emit a vocal response by displaying this instruction: "Please say the correct word". All recorded vocal responses were followed by the consequences described above. This block of trials continued until each target response was registered as correct during at least four out of the last five respective prompts.

Simple-discrimination trials. Each of these trials began by presenting a yellow empty square centered on the screen. A stimulus with a white background appeared inside the yellow square after a random delay ranging from 0 to $3 \mathrm{~s}$. The delay reset to $5 \mathrm{~s}$ if a click of the mouse or one of the target vocal responses was registered before the stimulus appeared (see Carlin, Wirth, \& Chase, 1998). Differential consequences were delivered as during acquisition of target responses. If nondifferential consequences were programmed, an empty screen appeared for $1.5 \mathrm{~s}$ after each registered vocal response. All correct responses were registered by the computer and added to each subject's earnings. Stimuli were presented randomly except that any stimulus was presented consecutively on no more than two trials and stimuli from the same set of simultaneously trained stimuli was presented equally often within a block of trials.

Baseline training. Each subject completed a minimum of 11 blocks of baseline training. Training of simple discriminations involving vocal responses was conducted via a delayed-prompt procedure (cf., Touchette, 1971). Every time a new discrimination was introduced, a prompt 
describing the correct response (e.g., "Remember to say 'Gox' to make a correct response”) was shown $3 \mathrm{~s}$ after the corresponding antecedent stimulus appeared. The prompt was not displayed if a target vocal response was registered before the delay elapsed. Differential consequences followed these responses as described above for the acquisition of target responses. Correct responses emitted following the prompt, however, did not yield any earnings for the subject. Instead, the message: "Too slow to earn money for your gift certificate" appeared after the selected logo was shown. Prompts were removed after one block of trials with the 3-s delay was completed with at least $90 \%$ correct responding.

As detailed in Table 1, baseline simple discriminations were trained in four stages. These discriminations related the $\mathrm{A}, \mathrm{B}$, and $\mathrm{C}$ stimuli and their respective target responses (hereafter designated as AR, BR, and CR discriminations). Each stage of baseline training was presented until the mastery criterion was met. The initial stage of baseline training presented the AR discriminations (A1Mek, A2Ruq, and A3Tib). The second stage trained the BR discriminations (B1Mek, B2Ruq, and B3'Tib) and the third stage taught the CR discriminations (C1Mek, C2Ruq, and C3Tib). During cumulative baseline training, all trained discriminations were intermixed. The mastery criterion required completing at least two consecutive blocks of trials with at least $90 \%$ correct responding for each set of trained discriminations. 
Table 1

Number of Training Trials per Block and Simple Discriminations Trained at Each Stage of Baseline Training for Functional Stimulus Classes in Experiment 1.

\begin{tabular}{lccccc}
\hline \multirow{2}{*}{ Training Stage } & $\begin{array}{c}\text { Trained Simple } \\
\text { Discriminations }\end{array}$ & AR & BR & CR & Total \\
\cline { 3 - 5 } 1. AR & A1Mek, A2Ruq, A3Tib & 36 & & 36 \\
2. BR & B1Mek, B2Ruq, B3Tib & & 36 & 36 \\
3. CR & C1Mek, C2Ruq, C3Tib & & & 36 \\
4. Cumulative & All of the above & 18 & 18 & 18 & 54 \\
Total & & 54 & 54 & 54 & 162 \\
\hline
\end{tabular}

Training of new responses required for transfer-of-function. This training was conducted to allow testing for emergent functional classes. Blocks consisted of 36 trials presenting one stimulus per class and one new target response. Specifically, simple discriminations A1Gox, A2Nal, and A3Vak were taught using differential consequences. Training followed the delayed-prompt procedure and mastery criterion specified for baseline training. Subsequently, differential consequences were reduced to $25 \%$ of the trials. A message describing this change in consequence presentation preceded each of these blocks of trials (Appendix E). All correct responses yielded earnings towards the gift certificate corresponding to the business logo assigned to this condition. Training of additional discriminations continued until mastery was demonstrated with reduced feedback presentation.

Testing for functional equivalence. A transfer-of-function test was performed to evaluate the emergence of functional stimulus classes. Blocks of testing consisted of 72 trials. Thirty-six trials 
presented the baseline trials A1Gox, A2Nal, and A3Vak; whereas the remaining 36 trials tested the emergence of the probe trials B1Gox, B2Nal, B3Vak; and C1Gox, C2Nal, C3Vak. These probes tested for transfer of each novel response to other members of the purported class. Baseline and probe trials were presented in random order. Tests for emergent discriminations appeared the same number of times within a block. Fifty percent of randomly selected baseline trials were followed by differential consequences; whereas probes were conducted in extinction. The message shown in Appendix E appeared before each block of testing. As before, all correct responses produced earnings for the gift certificate corresponding to the business logo shown as a consequence. Retraining occurred if baseline correct responding fell below $90 \%$ on any block of testing. Remedial training consisted of blocks of training of the new responses in the presence of A1, A2 and A3 stimuli. Remedial training continued until mastery was demonstrated. Afterwards, testing resumed. Testing continued until responding was stable. Stability criteria required the percent correct responding on probes for each of the last three testing blocks to vary no more than $10 \%$ from their overall mean. Absence of an upward or downward trend in correct responding to probes also was required. Additional remedial training was conducted if class-consistent probe responding was not observed. Remedial training consisted of blocks of baseline training followed by blocks of training of the new responses. Whenever necessary, prompts were presented during remedial training according to the procedures described above. Once this remedial training was completed, testing resumed. Additional cycles of remedial training and testing were conducted until stable classconsistent probe responding occurred or until it became apparent that accurate probe responding would not be forthcoming.

Equivalence Classes

Conditional-discrimination trials. Trials consisted of MTS tasks. Each of these trials began by presenting a red empty square. After a delay, a sample appeared in a white-background button 
located inside the red square. Contingencies governing the delay were identical to those of simplediscrimination trials. Clicking a visible sample resulted in presentation of four blue squares, one on each corner of the screen. Three different comparison stimuli were shown, each in a whitebackground button located inside one randomly selected blue square. A mouse click on a comparison stimulus registered a subject's selection for that trial. Clicking the correct comparison stimulus constituted a correct response. Clicking a comparison stimulus other than the correct comparison constituted an incorrect response. Correct responses were followed by class-specific consequences consisting of the logos for businesses preferred by the subject. These stimuli were presented for $1.5 \mathrm{~s}$, along with a distinctive tone that lasted $0.75 \mathrm{~s}$. Correct responses added one cent to the earnings corresponding to the displayed logo. Consequences for incorrect responses were delivered as described for the acquisition of target responses. If non-differential consequences were programmed, an empty screen appeared for $1.5 \mathrm{~s}$ after a comparison stimulus was selected. All correct responses were registered by the computer and added to each subject's earnings. MTS trials were presented randomly except that the same sample stimuli were presented consecutively a maximum of two trials, stimuli from the same stimulus set were presented equally often within a block of trials, and the correct comparison stimulus was shown in the same position no more than two trials.

MTS pretraining. Pretraining blocks lasted 26 trials. Trials consisted of matching an upper case English letter to its lower case equivalent (e.g., matching "A" to "a"). This block began with two demonstration trials performed by the experimenter. In the first demonstration trial a correct comparison was selected; whereas in the second demonstration an incorrect comparison was chosen. Differential consequences were arranged. A summary of the contingencies in effect was displayed on the screen after each demonstration trial (Appendix F). The subject completed the remaining 24 trials without any further instruction. The letter to be presented as sample for each trial 
was selected randomly without replacement. Pretraining continued until $90 \%$ correct responding was attained in a block of trials. All subjects required only one block of pretraining to reach this criterion. Baseline training. Each subject completed a minimum of 8 blocks of baseline conditional discrimination training. Baseline training featured class-specific reinforcers and differential consequences aimed at teaching two sets of three conditional discriminations with stimuli from the second stimulus set. The following discriminations were trained: D1E1, D2E2, D3E3; and E1F1, E2F2, E3F3. As shown in Table 2, baseline training consisted of four stages. Conditional discriminations were trained one set at a time, beginning with the DE discriminations. Once mastery of this set of discriminations was demonstrated, the EF discriminations were trained. Afterwards, cumulative baseline training presented all previously trained conditional discriminations intermixed. Once responding reached criterion, blocks of final baseline training were completed. These blocks featured all baseline conditional discriminations with differential consequences being randomly presented during only $25 \%$ of the trials. As described above for training of new responses for transfer-of-function tests, a message describing this change in consequence presentation preceded each of these blocks of trials (Appendix E). All correct responses yielded earnings towards the gift certificate specific to each purported class. The mastery criterion specified for training of functional stimulus classes was followed in this condition. 
Table 2

Number of Training Trials per Block and Conditional Discriminations Trained at Each Stage of Baseline Training for Equivalence Classes in Experiment 1.

\begin{tabular}{lcccc}
\hline \multirow{2}{*}{ Training Stage } & $\begin{array}{c}\text { Trained Conditional } \\
\text { Discriminations }\end{array}$ & \multicolumn{3}{c}{ Number of Trials per Block } \\
\cline { 3 - 5 } & D1F1, D2F2, D3F3 & 36 & EF & Total \\
\hline \hline 1. DF & E1F1, E2F2, E3F3 & & 36 & 36 \\
3. Cumulative & All of the above & 27 & 27 & 54 \\
4. Final & All of the above & 36 & 36 & 72 \\
Total & & 99 & 99 & 198 \\
\hline
\end{tabular}

Testing for stimulus equivalence. Testing blocks of 72 trials followed baseline training. Forty-two trials presented previously trained discriminations (baseline trials). The remaining 30 trials showed probes for emergent symmetric and combined relations, as well as emergent conditional discriminations involving the selected business logos presented either as samples or comparisons (see Table 3). These probes for emergent conditional discriminations tested whether class-specific consequences became members of the purported equivalence classes. Each probe was presented once per block of testing. The message shown in Appendix E preceded each block of this condition. Testing continued until responding was stable. Procedures for baseline and probe presentation, presentation of differential consequences, completion of remedial training, and stability criteria were to follow the procedures for testing for functional equivalence. Class-specific logos, however, were presented during baseline trials in which differential consequences were scheduled. All correct 
responses produced earnings for the gift certificates specific to each class. Remedial training consisted of blocks of cumulative and final baseline training.

Table 3

Probes for Emergent Relations in Experiment 1 after Baseline Training for Equivalence Classes.

\begin{tabular}{ccc}
\hline $\begin{array}{c}\text { Conditional } \\
\text { Discriminations }\end{array}$ & Symmetry & Combined \\
\hline D1G1, D2G2, D3G3 & F1D1, F2D2, F3D3 & D1E1, D2E2, D3E3 \\
E1G1, E2G2, E3G3 & F1E1, F2E2, F3E3 & E1D1, E2D2, E3D3 \\
F1G1, F2G2, F3G3 & \\
G1D1, G2D2, G3D3 & \\
G1E1, G2E2, G3E3 & \\
G1F1, G2F2, G3F3 & \\
\hline
\end{tabular}

Additional testing for functional equivalence. Subjects who demonstrated stable class-consistent responding during testing for stimulus equivalence resumed testing for functional class formation. These blocks of testing followed the same procedure set during initial testing for functional equivalence. This testing stage was conducted to ensure that class-consistent responding for the independent functional and equivalence classes was observed immediately before proceeding with the merging of arbitrary classes.

\section{Results}

\section{Experimental Mortality}

Of the nine subjects who participated in this experiment, five withdrew from the study prior to being tested for emergence of both functional and equivalence classes. All of these subjects expressed problems related to time constraints in their personal schedules. Of the four remaining 
subjects, three were dismissed after failing to demonstrate or maintain class-consistent responding for either the functional or equivalence classes. The last subject finished testing for the independent functional and equivalence classes, but then withdrew from the experiment because of time constraints. Data are reported only for the four subjects who reached testing for emergent classes. Baseline Training

All subjects who received baseline training for function equivalence successfully acquired the targeted discriminations. Table 4 presents the number of blocks of trials completed by each subject in order to demonstrate mastery at each stage of baseline training for the functional stimulus classes. Subjects 412, 718, and 315 successfully completed all of these stages of baseline training. Because Subject 851 began her participation with training and testing for equivalence classes and failed to demonstrate stimulus equivalence, she was dismissed from the study. Consequently, she did not receive baseline training for functional stimulus classes. 
Table 4

Blocks of Trials completed by each Subject at each stage of Baseline Training for Functional Stimulus Classes in Experiment 1.

\begin{tabular}{lccc}
\hline \multicolumn{1}{c}{ Training Stage } & Subject 412 & Subject 718 & Subject 315 \\
\hline AR Training / Prompts & 2 & 2 & 3 \\
AR Training / No Prompts & 2 & 2 & 2 \\
BR Training / Prompts & 2 & 2 & 2 \\
BR Training / No Prompts & 2 & 2 & 2 \\
CR Training / Prompts & 1 & 2 & 2 \\
CR Training / No Prompts & 2 & 2 & 2 \\
Cumulative Training & 9 & 2 & 2 \\
New Responses / Prompts & 3 & 2 & 2 \\
New Responses/No Prompts & 2 & 2 & 2 \\
New Responses/Reduced Density & 2 & 2 & 2 \\
Total Blocks of Trials & 27 & 2 & 2 \\
\hline
\end{tabular}

All subjects who received baseline equivalence training successfully acquired the conditional discriminations. Table 5 presents the respective number of blocks of trials completed by each subject before demonstrating mastery at each stage of baseline training for the equivalence classes. Subjects 412, 851, and 315 successfully completed baseline equivalence training. Because Subject 718 began her participation with functional stimulus classes and failed to demonstrate functional equivalence, she was dismissed from the experiment. Consequently, she did not receive stimulus equivalence training. 
Table 5

Blocks of Trials completed by each Subject at each stage of Baseline Training for Equivalence Classes in Experiment 1.

\begin{tabular}{lccc}
\hline \multicolumn{1}{c}{ Training Stage } & Subject 412 & Subject 851 & Subject 315 \\
\hline \hline DF Training & 4 & 4 & 3 \\
EF Training & 2 & 2 & 2 \\
Cumulative Training & 4 & 2 & 2 \\
Final Training & 2 & 2 & 2 \\
Total Blocks of Trials & 10 & 8 & 7 \\
\hline
\end{tabular}

\section{Baseline Retraining}

Subjects received remedial training when they failed to demonstrate class-consistent responding during testing for either functional or equivalence classes. Table 6 presents the number of blocks of trials completed by each subject at each stage of baseline retraining for functional equivalence. Retraining was arranged for Subjects 718 and 315, who showed mastery of all targeted baseline discriminations. Data for Subject 412 are not available because she withdrew from the experiment before necessary baseline retraining could be provided. As noted above, results for Subject 851 are not provided because this student did not participate in training for the functional classes. 
Table 6

Blocks of Trials completed by each Subject at each stage of Baseline Retraining for Functional Stimulus Classes in Experiment 1.

\begin{tabular}{lcc}
\hline \multicolumn{1}{c}{ Training Stage } & Subject 718 & Subject 315 \\
\hline \hline Cumulative Training / Prompts & 4 & 2 \\
Cumulative Training / No Prompts & 1 & 3 \\
New Responses / Prompts & 4 & 2 \\
New Responses/No Prompts & 1 & 1 \\
New Responses/Reduced Density & 2 & 1 \\
Total Blocks of Trials & 11 & 9 \\
\hline
\end{tabular}

Subject 851 required baseline retraining for the equivalence classes because of deficient classconsistent probe responding (see below). She completed one block each of cumulative and final training. This student maintained mastery of the targeted baseline discriminations throughout remedial training. Subjects 412 and 315 did not require baseline retraining for the equivalence classes. As noted earlier, Subject 718 was dismissed from the experiment before completing any training for the equivalence classes.

Testing for Independent Functional and Equivalence Classes

Subject 412 showed highly accurate baseline and probe responding during initial testing for both functional and equivalence classes. Figure 5 shows successive blocks of testing for functional equivalence for Subject 412; whereas Figure 6 depicts this subject's corresponding performance during testing for stimulus equivalence. 


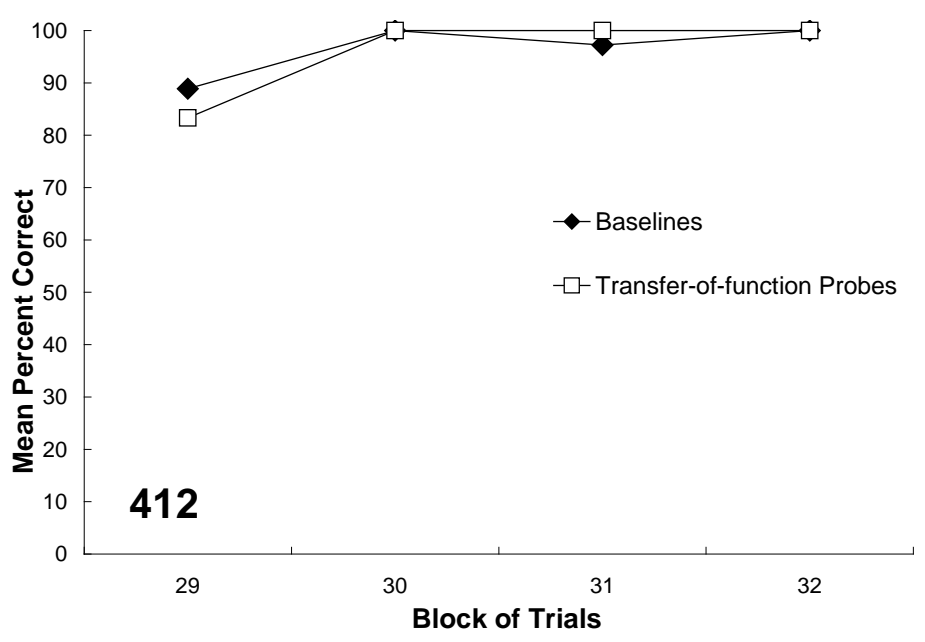

Figure 5. Mean percent correct responding for baseline and probe trials during initial testing for functional equivalence for Subject 412.

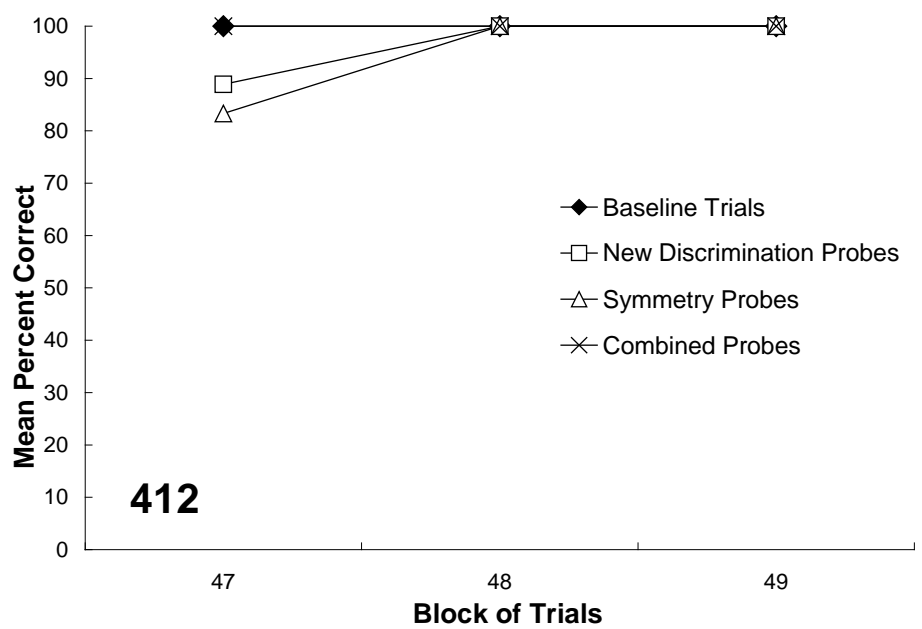

Figure 6. Mean percent correct responding for baseline and probe trials during testing for stimulus equivalence for Subject 412.

In spite of accurate responding during initial testing for functional and equivalence classes, when Subject 412 was tested again for emergent functional classes she failed to maintain classconsistent performance. Figure 7 shows her performance on baseline and probe trials and, despite $86 \%$ to $100 \%$ performance on baselines trials, probe performance remained below $70 \%$. As stated previously, this subject finished her participation in the experiment at this point. 


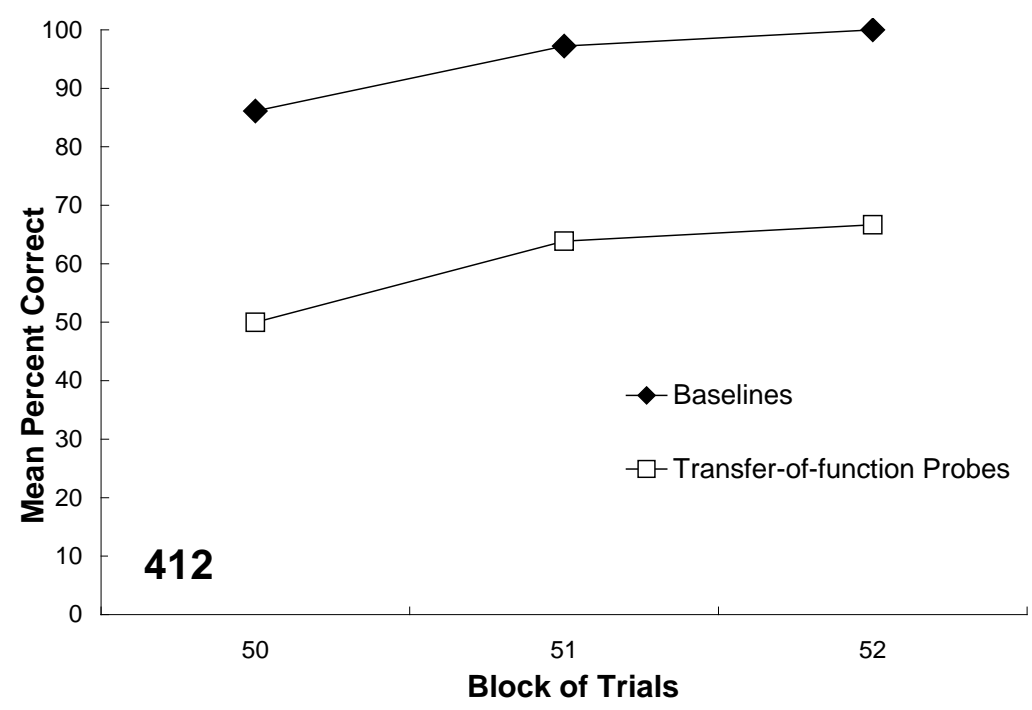

Figure 7. Mean percent correct responding for baseline and probe trials during additional testing for functional equivalence for Subject 412.

In contrast to Subject 412's performance, Subject 718 failed to demonstrate emergent functional classes. Even though Subject 718 successfully demonstrated highly accurate baseline performance across repeated blocks of testing, Figure 8 shows complete absence of accurate probe responding throughout successive blocks of testing for functional equivalence.

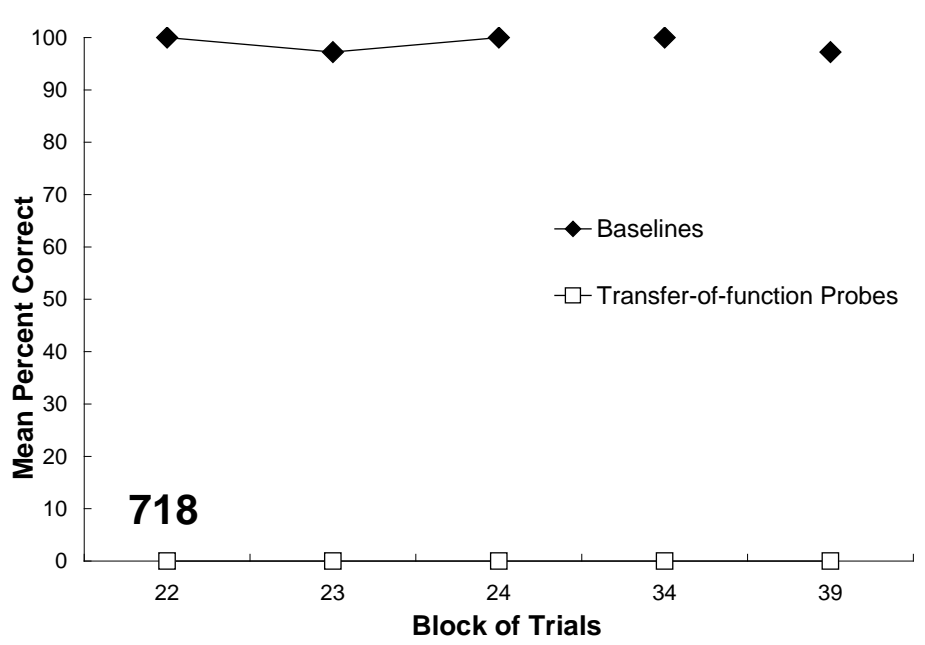

Figure 8. Mean percent correct responding for baseline and probe trials during testing for functional equivalence for Subject 718. Breaks in data series indicate instances in which testing was suspended to conduct remedial training. 
As noted above, Subject 851 did not complete either blocks of training or testing for functional classes. Nonetheless, as depicted in Figure 9, Subject 851 showed consistently accurate baseline responding across successive blocks of testing for stimulus equivalence. Responding by this student during symmetry and combined probes became increasingly accurate across blocks of testing, until accuracy levels comparable to baseline performance were reached. Subject 851, however, failed to demonstrate class-consistent responding during probes consisting of new discriminations in which stimuli arranged as class-specific reinforcers during baseline training were presented as antecedent stimuli. Accuracy for these probes remained consistently low even after Subject 851 completed all required baseline retraining.

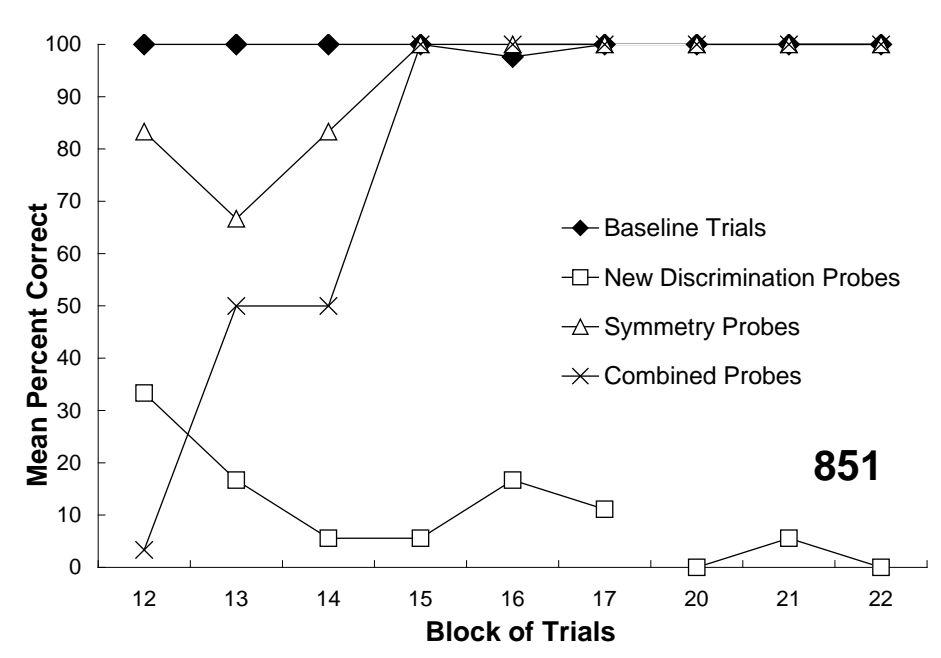

Figure 9. Mean percent correct responding for baseline and probe trials during testing for stimulus equivalence for Subject 851. Breaks in data series indicate instances in which testing was suspended to conduct remedial training.

Subject 315 showed deficient probe performance when testing for functional classes was first introduced. As shown in Figure 10, probe responding by this participant remained mostly below $80 \%$ across successive blocks of testing until remedial training was provided. After remedial 
training, this student demonstrated stable class-consistent baseline and probe responding when testing for functional classes resumed.

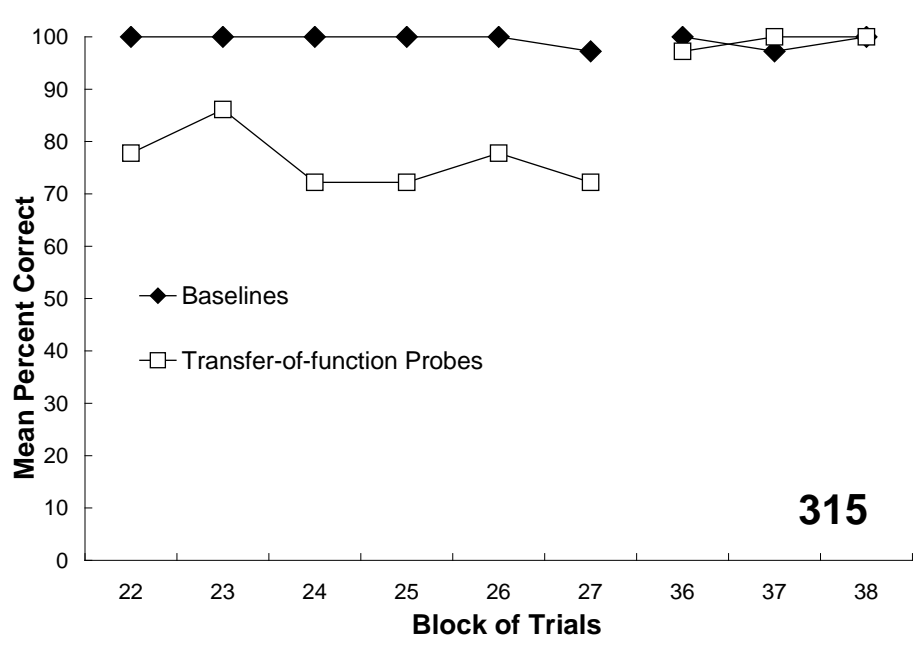

Figure 10. Mean percent correct responding for baseline and probe trials during initial testing for functional equivalence for Subject 315. Breaks in data series indicate instances in which testing was suspended to conduct remedial training.

In contrast, Subject 315 readily demonstrated stable class-consistent responding during testing for equivalence classes. Figure 11 depicts this student's performance across successive blocks of testing for stimulus equivalence. 


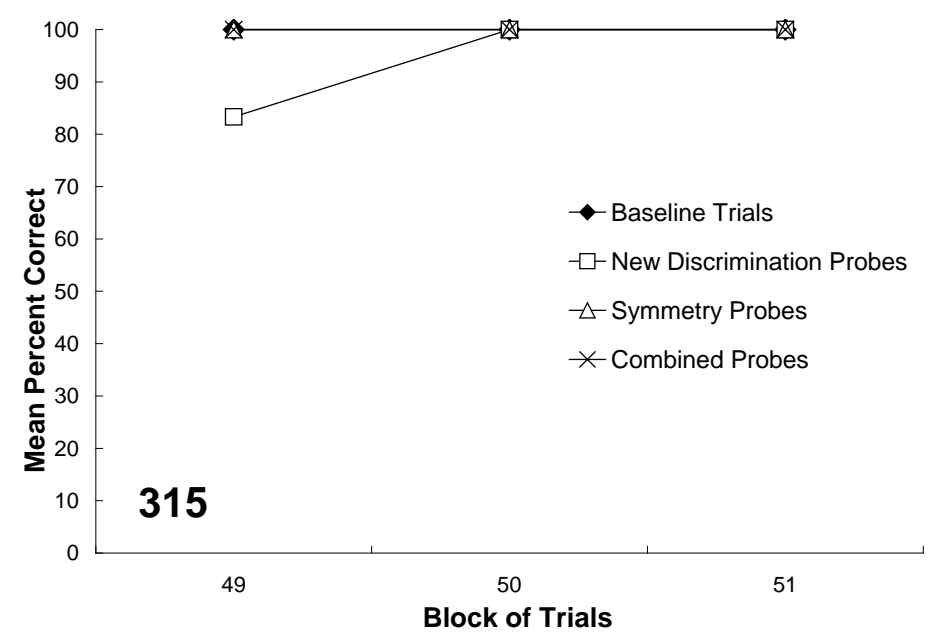

Figure 11. Mean percent correct responding for baseline and probe trials during testing for stimulus equivalence for Subject 315. Breaks in data series indicate instances in which testing was suspended to conduct remedial training.

After demonstrating stimulus equivalence, Subject 315 was retested for functional classes. As depicted in Figure 12, this student maintained class-consistent baseline and probe responding across successive blocks of testing. This participant, however, finished her participation in the experiment at this point due to time constrains.

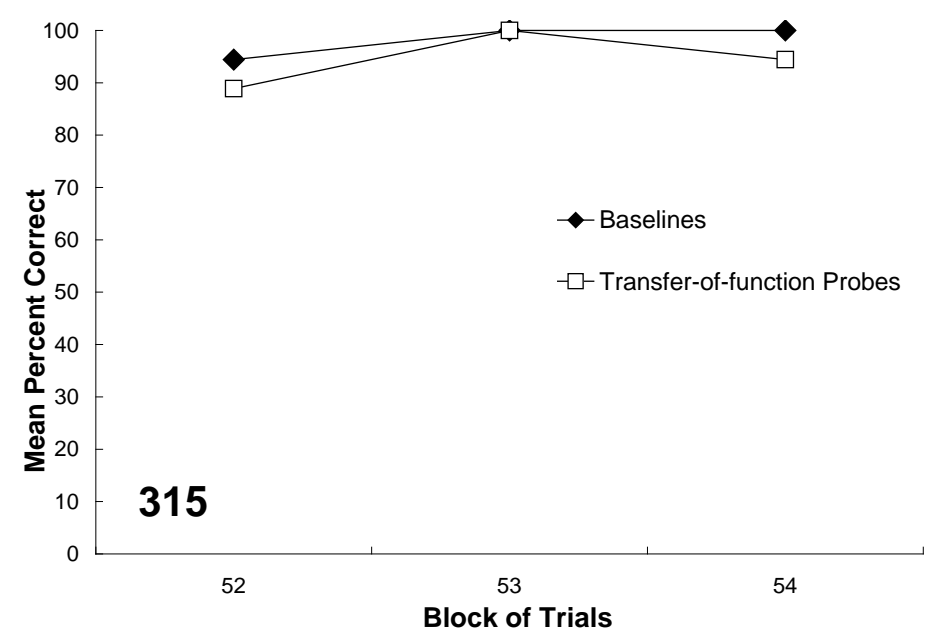

Figure 12. Mean percent correct responding for baseline and probe trials during additional testing for functional equivalence for Subject 315. 


\section{Discussion}

All subjects mastered the baseline discriminations that previous research had suggested would be sufficient to demonstrate emergence of independent functional and equivalence classes (e.g., Dube et al., 1987; Wirth \& Chase, 2002). Accordingly, responding by Subjects 412 and 315 suggested the formation of both types of stimulus classes. Nonetheless, class-consistent emergent responding was not observed for Subjects 718 and 851, who showed deficient correct responding during probes for functional and stimulus equivalence, respectively. In addition, Subject 412 did not maintain accurate probe performance during additional testing for functional classes.

Remedial training also was not effective in producing class-consistent emergent responding in most cases. Although all subjects demonstrated mastery of the baseline discriminations taught during remedial training, only Subject 315 subsequently demonstrated the targeted independent functional and equivalence classes. This participant, however, left the experiment before training for class merging could be conducted.

Consequently, Experiment 1 failed to provide the conditions necessary to assess class union. Even though previous studies have produced emergent responding with similar procedures (e.g., Dube et al., 1987; Wirth \& Chase, 2002), these studies did not arrange for the same individuals to complete training and testing for both functional and stimulus equivalence with different stimulus sets. As in the case of the current experiment, research combining these procedures has yielded limited results (e.g., Chase et al., 2008).

A few procedural variables may have prevented Experiment 1 from achieving its goal of systematically manipulating ordered pairs relating independent functional and equivalence classes. First, baseline training for functional classes was relatively lengthy. At least 20 blocks of trials were required for subjects to complete this training condition, in contrast with a maximum of 10 blocks of trials required for subjects to complete training for equivalence classes. Remedial functional 
training also comprised 9 and 11 blocks of trials for the two participants who were exposed to this type of retraining. The length of these conditions suggests that achieving mastery of the number of discriminations taught was difficult for these subjects and this may have contributed to failures to demonstrate and maintain class-consistent performance during probes for functional equivalence.

The use of class-specific consequences during training for equivalence classes also may have added a level of complexity to testing for stimulus equivalence. In the case of Subject 851, correct responding was erratic during probes in which class-specific consequences were presented as discriminative or conditional stimuli. Using class-specific consequences would contribute as well to the complexity of testing for merged classes by increasing significantly the number of possible probes for emergent discriminations.

In addition to these procedural considerations, the complexity of the current experiment for the subjects may be related to the integration of two different types of verbal behavior (Michael, 1985). According to Michael, the conditional discriminations required for stimulus equivalence exemplify selection-based verbal behavior in which reinforcement is delivered upon selection of a particular stimulus from a range of available stimuli. In contrast, the simple discriminations required for functional equivalence involve topography-based verbal behavior in which a stimulus controls the occurrence of a response characterized by its distinguishable topography. Consequently, occurrence of class-consistent responding may have been hampered by requiring subjects to respond in these two different ways in the presence of abstract stimuli that were highly similar and have a limited history of reinforcement.

The limitations in Experiment 1 might be addressed via procedural changes. Baseline training for functional classes can be expedited by reducing the number of simple discriminations required to produce functional equivalence. An emergent functional class is demonstrated usually by testing for transfer-of-function from one stimulus to another. Thus, if two stimuli have been 
previously related (e.g., by a training a conditional discrimination), only one simple discrimination featuring one of these stimuli needs to be trained to test for control of the trained response in the presence of the remaining stimulus.

Additionally, using class-specific consequences during baseline training for equivalence classes is not required to demonstrate stimulus equivalence. Removing these consequences limits the number of possible emergent relations that can be tested, but will diminish the complexity of the stimulus classes and facilitate the occurrence of class-consistent emergent responding. Equivalence classes also can be trained and tested first. As noted earlier, remedial training and subsequent testing for equivalence classes required a relatively short number of blocks of trials. Thus, delays before proceeding to class merging would be reduced in instances in which equivalence retraining is necessary after functional classes have been demonstrated.

\section{Experiment 2}

This experiment addressed the difficulties of Experiment 1 in arranging for subjects to reliably demonstrate independent functional and equivalence classes. As in Experiment 1, this experiment was concerned with arranging class union via training of ordered pairs consisting of simple or conditional discriminations. Experiment 2 relied on two different types of ordered pairs to produce merging of classes: a) an antecedent stimulus from an equivalence class was trained as a discriminative stimulus for a response controlled by a functional class, and b) a stimulus from an equivalence class was related with a stimulus from a functional class via a conditional discrimination.

The general design for this experiment is presented in Figure 13. Experiment 2 began by provided MTS pretraining to all participants. Subsequently, subjects completed training and testing for equivalence classes. Subjects then were taught the target vocal responses, which were used in training and testing for functional stimulus classes. Additional testing for equivalence and functional classes was conducted to establish that independent classes were maintained prior to class merging. 
For Subjects 452 and 518, training for class merging was conducted via simple discriminations relating the responses controlled by the functional classes to the set of stimuli $\mathrm{A}$ from the equivalence classes. Subjects 271 and 807 completed training for class merging via the AE set of conditional discriminations. Training for class expansion followed comprehensive testing for merging of classes. Training for class expansion consisted of the FE set of conditional discriminations for those participants (Subjects 452 \& 518) whose training for class merging consisted of simple discriminations. Conversely, Subjects 271 and 817 completed training for class merging in which simple discriminations linking that target responses to novel stimuli $\mathrm{F}$ were presented. All subjects finished their participation in this experiment by undergoing testing for class expansion. What follows is a detailed description of the methods used in Experiment 2. 


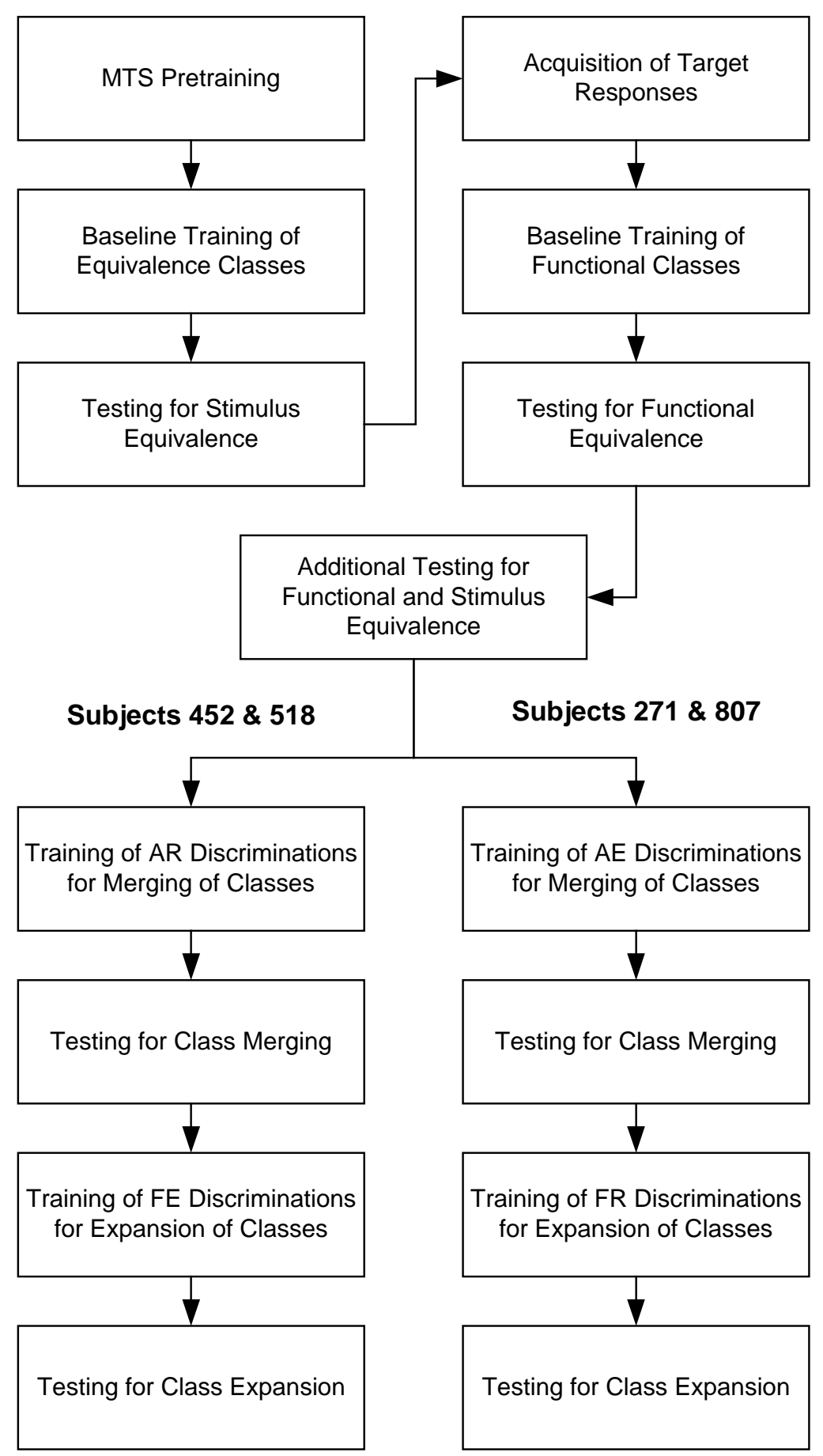

Figure 13. Schematic diagram of the general design for Experiment 2. 


\section{Method}

Subjects, Apparatus and Setting

Nine female WVU undergraduate students participated in this experiment. Procedures for subject recruitment were identical to those of Experiment 1. Compensation for research participation was conducted in a manner similar to Experiment 1. In Experiment 2, however, subjects were asked at the end of their participation whether they wanted to receive their monetary earnings in the form of gift certificates or a personal check. All subjects chose the latter. The apparatus and setting for this experiment were identical to those of Experiment 1

Stimuli and Target Vocal Responses

The 18 abstract figures used in Experiment 1 were adopted for this experiment. Figure 14 shows all stimuli used in Experiment 2. Stimulus set 1 was used for establishing the equivalence classes; whereas stimulus set 2 was used for the functional stimulus classes. As shown in Figure 14, one additional stimulus per class was used to expand each merged class. Stimuli were labeled following the notation system described in Experiment 1. Subjects did not have access to this nomenclature. Figure 14 also shows the three nonsense vocal responses targeted in Experiment 2. 


\section{Stimulus Set 1}

1

句

C
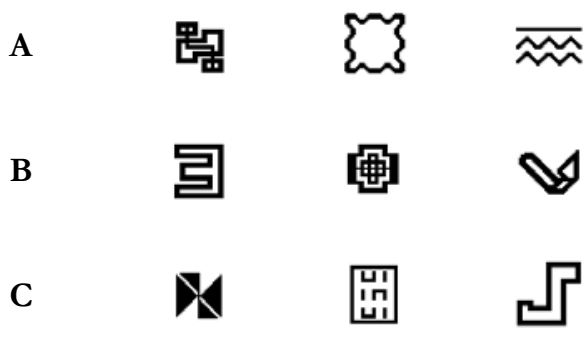

2

串

प्रा

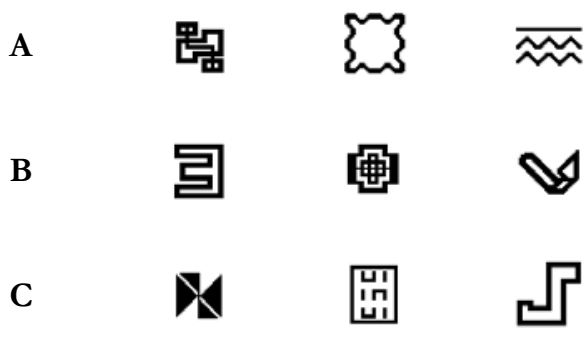

3

Stimulus Set 2

1

2

3

D

E

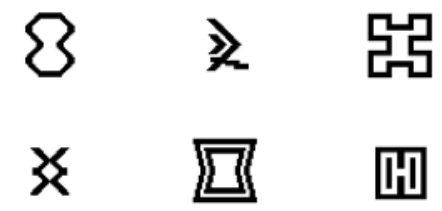

\section{Class Expansion}

1

2

3

F
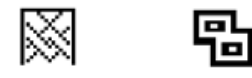

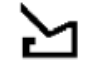

\section{Target Responses}

1

2

3

Rsp Mek Ruq Tib

Figure 14. Stimuli and target responses used in Experiment 2. Sets of stimuli A through F correspond to the stimuli used during training of functional and equivalence classes for Experiment 2. The target responses refer to class-specific responses used in Experiment 2. 
Equivalence Classes

Conditional-discrimination trials. These trials consisted of MTS tasks, which followed the procedures described in Experiment 1, except that when differential consequences were arranged, correct responses were followed by presenting a green check mark $(\checkmark)$ for $1.5 \mathrm{~s}$, along with a distinctive tone that lasted $0.75 \mathrm{~s}$. All other consequences were presented as described in Experiment 1.

MTS pretraining and baseline training. Pretraining and baseline training were conducted following the procedures established in Experiment 1. Demonstration trials during MTS pretraining, however, were followed by the respective description of the contingencies in effect as shown in Appendix G. Baseline training was conducted in four stages. Table 7 shows the targeted conditional discriminations and their respective number of trials per block at each stage of baseline training.

Table 7

Number of Training Trials per Block and Conditional Discriminations Trained at Each Stage of Baseline Training for Equivalence Classes in Experiment 2.

\begin{tabular}{lcccc}
\hline & & \multicolumn{3}{c}{ Number of Trials per Block } \\
\cline { 3 - 4 } Training Stage & $\begin{array}{c}\text { Trained Conditional } \\
\text { Discriminations }\end{array}$ & AC & BC & Total \\
\hline 1. AC & A1C1, A2C2, A3C3 & 36 & 36 & 36 \\
2. BC & B1C1, B2C2, B3C3 & & 27 & 54 \\
3. Cumulative & All of the above & 27 & 36 & 72 \\
4. Final & All of the above & 36 & 99 & 198 \\
Total & & 99 & & 36 \\
\hline
\end{tabular}


Testing for stimulus equivalence. Testing blocks of 72 trials followed baseline training. Thirty-six trials consisted of previously trained discriminations (baseline trials). The remaining 36 trials showed probes for emergent symmetric and combined relations (see Table 8). Blocks of testing followed all other procedures described in Experiment 1.

Table 8

Baseline Trials and Probes for Emergent Relations presented during Blocks of Testing for Equivalence Classes in Experiment 2.

\begin{tabular}{ccc}
\hline Baseline Trials & Symmetry & Probes \\
& C1A1, C2A2, C3A3 & A1B1, A2B2, A3B3 \\
\hline A1C1, A2C2, A3C3 & C1B1, C2B2, C3B3 & B1A1, B2A2, B3A3 \\
\hline
\end{tabular}

Functional Stimulus Classes

Acquisition of target responses. As in Experiment 1, prior to training the functional classes, a block of trials was completed to identify accurately each subject's utterances of all target responses. These responses were trained according to the procedures set for Experiment 1; except that correct responses were followed by a green check mark.

Simple-discrimination trials. These trials followed the procedures established for Experiment 1, except that, as in previous conditions in the current experiment, a green check mark followed correct responses when differential consequences were arranged.

Baseline training. Each subject completed a minimum of eight blocks of baseline training. Baseline training comprised simple and conditional discriminations. As shown in Table 9, baseline discriminations were trained in four stages. Each stage was presented until the corresponding 
mastery criterion set in Experiment 1 was met. The initial stage of baseline training taught the DE conditional discriminations (D1E1, D2E2, and D3E3) and the second stage trained the ER simple discriminations (E1Mek, E2Ruq, and E3Tib). During cumulative baseline training, all trained discriminations were intermixed. The final stage of baseline training featured all targeted discriminations with differential consequences being randomly arranged for only $25 \%$ of the trials.

Table 9

Number of Training Trials per Block and Simple Discriminations Trained at Each Stage of Baseline Training for Functional Stimulus Classes in Experiment 2.

\begin{tabular}{lcccc}
\hline \multirow{2}{*}{ Training Stage } & $\begin{array}{c}\text { Trained Stimulus } \\
\text { Discriminations }\end{array}$ & DE & ER & Total \\
\cline { 3 - 5 } 1. DE & D1E1, D2E2, D3E3 & 36 & 36 & 36 \\
2. ER & E1Mek, E2Ruq, E3Tib & & 27 & 36 \\
3. Cumulative & All of the above & 27 & 36 & 72 \\
4. Final & All of the above & 36 & 99 & 198 \\
Total & & 99 & 99 & \\
\hline
\end{tabular}

Testing for functional equivalence. Blocks of testing consisted of 72 trials. Thirty-six trials presented baseline trials; whereas the remaining 36 trials consisted of probes. Half of the probes assessed whether functional stimulus classes emerged via a transfer-of-function test. The rest of the probes tested for emergent symmetric relations for the baseline conditional discriminations. Table 10 shows all presented baseline and probe trials. All other procedures for trial presentation were the same of Experiment 1. Retraining occurred if baseline correct responding fell below 90\% on any 
block of testing. Remedial training consisted of blocks of final baseline training. Remedial training continued until mastery was demonstrated. Afterwards, testing resumed.

Table 10

Baseline Trials and Probes for Emergent Relations presented during Blocks of Testing for Functional Classes in Experiment 2 .

\begin{tabular}{ccc}
\hline Baseline Trials & Pymmetry & Simple Discriminations \\
\hline \hline D1E1, D2E2, D3E3 & E1D1, E2D2, E3D3 & D1Mek, D2Ruq, D3Tib \\
E1Mek, E2Ruq, E3Tib & & \\
\hline
\end{tabular}

\section{Additional Testing for Functional and Stimulus Equivalence}

Subjects who demonstrated stable accurate responding during testing for functional equivalence resumed testing for stimulus equivalence. Additional alternations between testing for functional and stimulus equivalence were conducted if several days elapsed since the last time maintenance of either functional or equivalence classes was assessed. Blocks of testing were conducted as specified previously in this experiment. Baseline and probe performance above $90 \%$ correct responding during one block of testing was required to assert maintenance of either type of emergent class. Additional blocks of testing for the same type of class were delivered for subjects whose participation in the experiment was interrupted for several days. Testing continued until stable responding was observed. The same stability criteria were followed as during testing for functional and stimulus equivalence. Extensive and repeated testing was conducted to demonstrate convincingly that independent functional and equivalence classes were demonstrated immediately prior to merging these arbitrary classes. 
Merging of Stimulus Classes

Baseline training. Training consisted of blocks of 36 trials. Differential consequences were arranged for all trails as described above. Training for Subjects 452 and 518 consisted of blocks of trials presenting the AR simple discriminations (A1Mek, A2Ruq, and A3Tib); whereas training for Subjects 271 and 807 presented the AE conditional discriminations (A1E1, A2E2, and A3E3). All other procedures were identical to those established for previous baseline training conditions in Experiment 2.

Testing for class merging. Blocks of testing consisted of 96 trials. Thirty-six trials consisted of baseline discriminations. Eighteen trials presented probes for all emergent discriminations tested prior to merging of stimulus classes. The remaining 42 trials presented probes for all novel emergent discriminations possible after training for class merging. For each block of testing, differential consequences were delivered for 24 randomly selected baseline trials; whereas all other trials were conducted in extinction. Table 11 shows all baseline and probe trials for each block of testing for Subjects 452 and 518. Table 12 shows the respective baseline and probe trials for Subjects 271 and 807. All other procedures for trial presentation and assessment of stable responding were identical to previous testing conditions in Experiment 2. 
Table 11

Baseline Trials and Probes for Emergent Relations presented to Subjects 452 and 518 during Blocks of Testing for Class Merging in Experiment 2.

\begin{tabular}{ccc}
\hline \multicolumn{3}{c}{ Baseline Trials } \\
\hline A1C1, A2C2, A3C3 & B1C1, B2C2, B3C3 & D1E1, D2E2, D3E3 \\
\hline E1Mek, A2Ruq, A3Tib & E1Mek, E2Ruq, E3Tib & \\
\hline A1B1, A2B2, A3B3 & B1A1, B2A2, B3A3 & C1A1, C2A2, C3A3 \\
C1B1, C2B2, C3B3 & E1D1, E2D2, E3D3 & D1Mek, D2Ruq, D3Tib \\
\hline Novel Emergent Discriminations tested after Merging Stimulus Classes \\
\hline A1D1, A2D2, A3D3 & A1E1, A2E2, A3E3 & B1D1, B2D2, B3D3 \\
B1E1, B2E2, B3E3 & C1D1, C2D2, C3D3 & C1E1, C2E2, C3E3 \\
D1A1, D2A2, D3A3 & D1B1, D2B2, D3B3 & D1C1, D2C2, D3C3 \\
E1A1, E2A2, E3A3 & E1B1, E2B2, E3B3 & E1C1, E2C2, E3C3 \\
B1Mek, B2Ruq, B3Tib & C1Mek, C2Ruq, C3Tib & \\
\hline
\end{tabular}


Table 12

Baseline Trials and Probes for Emergent Relations presented to Subjects 271 and 807 during Blocks of Testing for Class Merging in Experiment 2.

\begin{tabular}{ccc}
\hline \multicolumn{3}{c}{ Baseline Trials } \\
\hline A1C1, A2C2, A3C3 & A1E1, A2E2, A3E3 & B1C1, B2C2, B3C3 \\
\hline E1E1, D2E2, D3E3 & E1Mek, E2Ruq, E3Tib & \\
\hline A1B1, A2B2, A3B3 & B1A1, B2A2, B3A3 & C1A1, C2A2, C3A3 \\
C1B1, C2B2, C3B3 & E1D1, E2D2, E3D3 & D1Mek, D2Ruq, D3Tib \\
\hline Novel Emergent Discriminations tested after Merging Stimulus Classes \\
\hline \hline A1D1, A2D2, A3D3 & B1D1, B2D2, B3D3 & B1E1, B2E2, B3E3 \\
C1D1, C2D2, C3D3 & C1E1, C2E2, C3E3 & D1A1, D2A2, D3A3 \\
D1B1, D2B2, D3B3 & D1C1, D2C2, D3C3 & E1A1, E2A2, E3A3 \\
E1B1, E2B2, E3B3 & E1C1, E2C2, E3C3 & A1Mek, A2Ruq, A3Tib \\
B1Mek, B2Ruq, B3Tib & C1Mek, C2Ruq, C3Tib & \\
\hline
\end{tabular}

Expansion of Stimulus Classes

Baseline training. Training consisted of blocks of 36 trials with differential consequences being delivered for all trials as described above. Training for Subjects 452 and 518 consisted of blocks of trials presenting the FE simple discriminations (F1E1, F2E2, and F3E3); whereas training for Subjects 271 and 807 presented the FR conditional discriminations (F1Mek, F2Ruq, and F3Tib). All other procedures were identical to those of previous baseline training conditions in Experiment 2. 
Testing for class expansion. Blocks of testing consisted of 96 trials. Thirty-six trials consisted of baseline discriminations. Thirty trials presented randomly selected probes from those tested prior to the expansion of stimulus classes. The remaining 30 trials consisted of probes for all novel emergent discriminations possible after training for class expansion. Differential consequences were delivered for 24 randomly selected baseline trials, with all remaining block trials conducted in extinction. Table 13 presents all possible baseline and probe trials for each block of testing for Subjects 452 and 518 . Table 14 presents the respective baseline and probe trials for Subjects 271 and 807 . All other procedures for trial presentation and assessment of stable responding were identical to previous testing conditions in Experiment 2. 
Table 13

Baseline Trials and Probes for Emergent Relations presented to Subjects 452 and 518 during Blocks of Testing for Class Expansion in Experiment 2.

\begin{tabular}{|c|c|c|}
\hline \multicolumn{3}{|c|}{ Baseline Trials } \\
\hline $\mathrm{A} 1 \mathrm{C} 1, \mathrm{~A} 2 \mathrm{C} 2, \mathrm{~A} 3 \mathrm{C} 3$ & $\mathrm{~B} 1 \mathrm{C} 1, \mathrm{~B} 2 \mathrm{C} 2, \mathrm{~B} 3 \mathrm{C} 3$ & D1E1, D2E2, D3E3 \\
\hline F1E1, F2E2, F3E3 & A1Mek, A2Ruq, A3Tib & E1Mek, E2Ruq, E3Tib \\
\hline \multicolumn{3}{|c|}{ Emergent Discriminations tested prior to Expanding Stimulus Classes } \\
\hline $\mathrm{A} 1 \mathrm{~B} 1, \mathrm{~A} 2 \mathrm{~B} 2, \mathrm{~A} 3 \mathrm{~B} 3$ & A1D1, A2D2, A3D3 & A1E1, A2E2, A3E3 \\
\hline B1A1, B2A2, B3A3 & B1D1, B2D2, B3D3 & B1E1, B2E2, B3E3 \\
\hline C1A1, C2A2, C3A3 & C1B1, C2B2, C3B3 & C1D1, C2D2, C3D3 \\
\hline C1E1, C2E2, C3E3 & D1A1, D2A2, D3A3 & D1B1, D2B2, D3B3 \\
\hline D1C1, D2C2, D3C3 & E1A1, E2A2, E3A3 & E1B1, E2B2, E3B3 \\
\hline E1C1, E2C2, E3C3 & E1D1, E2D2, E3D3 & B1Mek, B2Ruq, B3Tib \\
\hline C1Mek, C2Ruq, C3Tib & D1Mek, D2Ruq, D3Tib & \\
\hline \multicolumn{3}{|c|}{ Novel Emergent Discriminations tested after Expanding Stimulus Classes } \\
\hline $\mathrm{A} 1 \mathrm{~F} 1, \mathrm{~A} 2 \mathrm{~F} 2, \mathrm{~A} 3 \mathrm{~F} 3$ & $\mathrm{~B} 1 \mathrm{~F} 1, \mathrm{~B} 2 \mathrm{~F} 2, \mathrm{~B} 3 \mathrm{~F} 3$ & $\mathrm{C} 1 \mathrm{~F} 1, \mathrm{C} 2 \mathrm{~F} 2, \mathrm{C} 3 \mathrm{~F} 3$ \\
\hline D1F1, D2F2, D3F3 & E1F1, E2F2, E3F3 & F1A1, F2A2, F3A3 \\
\hline F1B1, F2B2, F3B3 & $\mathrm{F} 1 \mathrm{C} 1, \mathrm{~F} 2 \mathrm{C} 2, \mathrm{~F} 3 \mathrm{C} 3$ & F1D1, F2D2, F3D3 \\
\hline F1Mek, F2Ruq, F3Tib & & \\
\hline
\end{tabular}


Table 14

Baseline Trials and Probes for Emergent Relations presented to Subjects 271 and 807 during Blocks of Testing for Class Expansion in Experiment 2.

\begin{tabular}{|c|c|c|}
\hline \multicolumn{3}{|c|}{ Baseline Trials } \\
\hline $\mathrm{A} 1 \mathrm{C} 1, \mathrm{~A} 2 \mathrm{C} 2, \mathrm{~A} 3 \mathrm{C} 3$ & A1E1, A2E2, A3E3 & B1C1, B2C2, B3C3 \\
\hline D1E1, D2E2, D3E3 & E1Mek, E2Ruq, E3Tib & F1Mek, F2Ruq, F3'Tib \\
\hline \multicolumn{3}{|c|}{ Possible Emergent Discriminations prior to Expanding Stimulus Classes } \\
\hline $\mathrm{A} 1 \mathrm{~B} 1, \mathrm{~A} 2 \mathrm{~B} 2, \mathrm{~A} 3 \mathrm{~B} 3$ & A1D1, A2D2, A3D3 & B1A1, B2A2, B3A3 \\
\hline B1D1, B2D2, B3D3 & B1E1, B2E2, B3E3 & C1A1, C2A2, C3A3 \\
\hline $\mathrm{C} 1 \mathrm{~B} 1, \mathrm{C} 2 \mathrm{~B} 2, \mathrm{C} 3 \mathrm{~B} 3$ & C1D1, C2D2, C3D3 & C1E1, C2E2, C3E3 \\
\hline D1A1, D2A2, D3A3 & D1B1, D2B2, D3B3 & D1C1, D2C2, D3C3 \\
\hline E1A1, E2A2, E3A3 & E1B1, E2B2, E3B3 & E1C1, E2C2, E3C3 \\
\hline E1D1, E2D2, E3D3 & A1Mek, A2Ruq, A3Tib & B1Mek, B2Ruq, B3Tib \\
\hline C1Mek, C2Ruq, C3Tib & D1Mek, D2Ruq, D3Tib & \\
\hline \multicolumn{3}{|c|}{ Possible Emergent Discriminations after Expanding Stimulus Classes } \\
\hline $\mathrm{A} 1 \mathrm{~F} 1, \mathrm{~A} 2 \mathrm{~F} 2, \mathrm{~A} 3 \mathrm{~F} 3$ & $\mathrm{~B} 1 \mathrm{~F} 1, \mathrm{~B} 2 \mathrm{~F} 2, \mathrm{~B} 3 \mathrm{~F} 3$ & $\mathrm{C} 1 \mathrm{~F} 1, \mathrm{C} 2 \mathrm{~F} 2, \mathrm{C} 3 \mathrm{~F} 3$ \\
\hline D1F1, D2F2, D3F3 & E1F1, E2F2, E3F3 & F1A1, F2A2, F3A3 \\
\hline F1B1, F2B2, F3B3 & $\mathrm{F} 1 \mathrm{C} 1, \mathrm{~F} 2 \mathrm{C} 2, \mathrm{~F} 3 \mathrm{C} 3$ & F1D1, F2D2, F3D3 \\
\hline F1E1, F2E2, F3E3 & & \\
\hline
\end{tabular}




\section{Results}

\section{Experimental Mortality}

Five of the nine subjects who participated in this experiment did not complete the study. Four of these subjects withdrew citing time constraints in their personal schedules. The last of these subjects was dismissed from the study after technical difficulties were found during testing for emerging classes. Data are reported only for the four subjects who were tested for merging and expansion of stimulus classes.

\section{Baseline Training for Equivalence and Functional Classes}

All subjects completed baseline training for stimulus equivalence and successfully acquired the targeted discriminations. Table 15 shows the number of blocks of trials completed by each subject in order to reach mastery at each stage of baseline training for the equivalence classes.

Table 15

Blocks of Trials completed by each Subject at each stage of Baseline Training for Equivalence Classes in Experiment 2.

\begin{tabular}{lcccc}
\hline \multicolumn{1}{c}{ Training Stage } & Subject 452 & Subject 518 & Subject 271 & Subject 807 \\
\hline \hline AC Training & 4 & 3 & 3 & 4 \\
BC Training & 3 & 2 & 2 & 2 \\
Cumulative Training & 2 & 2 & 2 & 2 \\
Final Training & 2 & 2 & 2 & 10 \\
Total Blocks of Trials & 11 & 9 & 9 & 2 \\
\hline
\end{tabular}


All subjects also successfully completed baseline training for functional stimulus classes.

Table 16 shows the number of blocks of trials completed by each subject in order to reach mastery at each stage of baseline training for the functional classes.

Table 16

Blocks of Trials completed by each Subject at each stage of Baseline Training for Functional Stimulus Classes in Experiment 2 .

\begin{tabular}{lcccc}
\hline \multicolumn{1}{c}{ Training Stage } & Subject 452 & Subject 518 & Subject 271 & Subject 807 \\
\hline \hline DE Training & 4 & 2 & 2 & 3 \\
ER Training / Prompts & 4 & 1 & 2 & 2 \\
ER Training / No Prompts & 3 & 2 & 2 & 2 \\
Cumulative Training & 4 & 2 & 2 & 2 \\
Final Training & 2 & 2 & 2 & 11 \\
Total Blocks of Trials & 17 & 9 & 10 & 2 \\
\hline
\end{tabular}

\section{Baseline Retraining for Equivalence and Functional Classes}

Subject 518 required retraining for equivalence classes because of deficient baseline accuracy during one block of testing for stimulus equivalence. Remedial training for this student consisted of one block of final training. Baseline performance by Subjects 452, 271, and 807 remained above mastery throughout all blocks of testing for stimulus equivalence. Thus, these students did not complete any retraining for equivalence classes.

Subjects 452 and 518 completed retraining for functional stimulus classes after their baseline accuracy fell below 90\% during blocks of testing. During testing for class expansion, Subject 807 reported difficulties with recognition of her utterances of the response "Tib" by the experimental 
application. Observations by the experimenter verified this assertion. Consequently, this student completed remedial training for functional classes in which the vocal response "Gox" replaced the response "Tib". The experimenter instructed this participant to say "Gox" for the rest of the experiment in every instance in which she had considered "Tib" to be the correct answer. Table 17 presents the number of blocks of trials completed by all subjects at each stage of remedial training. Subject 271 did not complete any retraining for functional classes because her baseline responding remained above mastery during all blocks of testing for functional equivalence.

Table 17

Blocks of Trials completed by Subjects 452, 518 and 807 at each stage of Baseline Retraining for Functional Stimulus Classes in Experiment 2.

\begin{tabular}{lccc}
\hline \multicolumn{1}{c}{ Training Stage } & Subject 452 & Subject 518 & Subject 807 \\
\hline \hline Cumulative Training / Prompts & 3 & --- & --- \\
Cumulative Training / No Prompts & 1 & --- & --- \\
Final Training / Prompts & --- & 1 & --- \\
Final Training / No Prompts & 1 & 1 & 1 \\
Total Blocks of Trials & 5 & 2 & 1 \\
\hline
\end{tabular}

Testing for Independent Functional and Equivalence Classes

All subjects successfully demonstrated the targeted independent functional and equivalence classes before merging of classes was conducted. Figure 15 shows all blocks of testing for stimulus equivalence for Subject 452; whereas Figure 16 depicts her respective responding during testing for functional equivalence. 


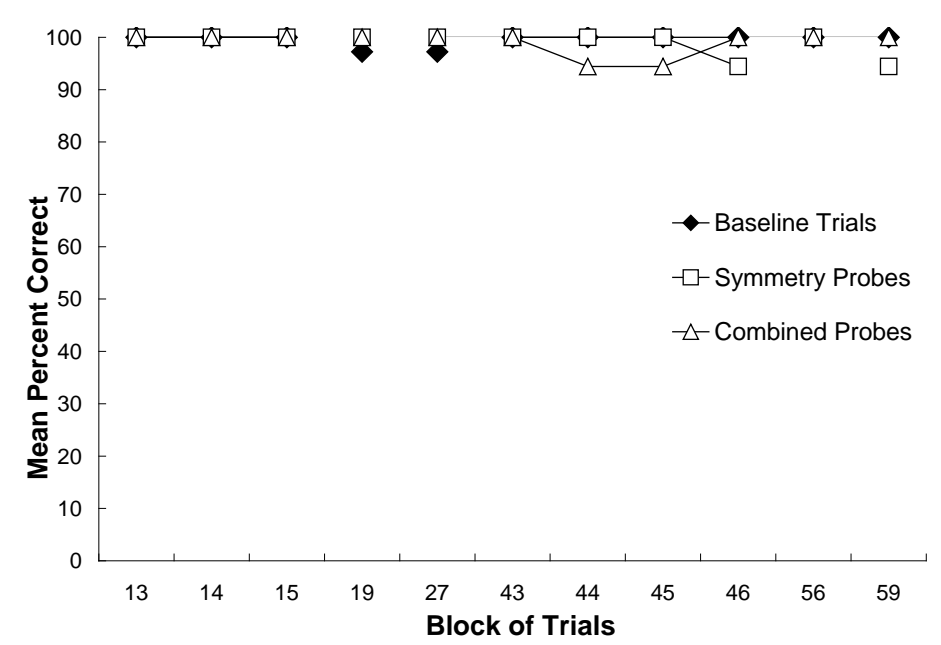

Figure 15. Mean percent correct responding for baseline and probe trials during testing for stimulus equivalence for Subject 452. Breaks in the data series indicate instances in which testing for stimulus equivalence alternated with testing for functional equivalence.

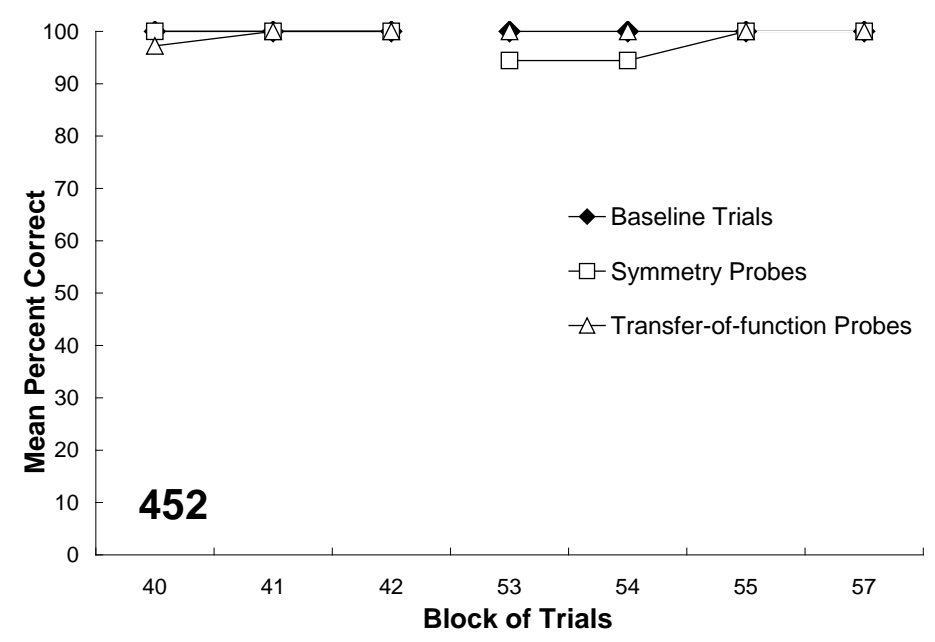

Figure 16. Mean percent correct responding for baseline and probe trials during testing for functional equivalence for Subject 452. Breaks in the data series indicate instances in which testing for functional equivalence alternated with testing for stimulus equivalence.

Figure 17 depicts all blocks of testing for stimulus equivalence for Subject 518, and Figure 18 shows her respective responding during testing for functional equivalence. Baseline and probe performance by Subject 518 fell below 90\% only in one block of testing each for functional and equivalence classes. Otherwise, this participant showed highly accurate responding. 


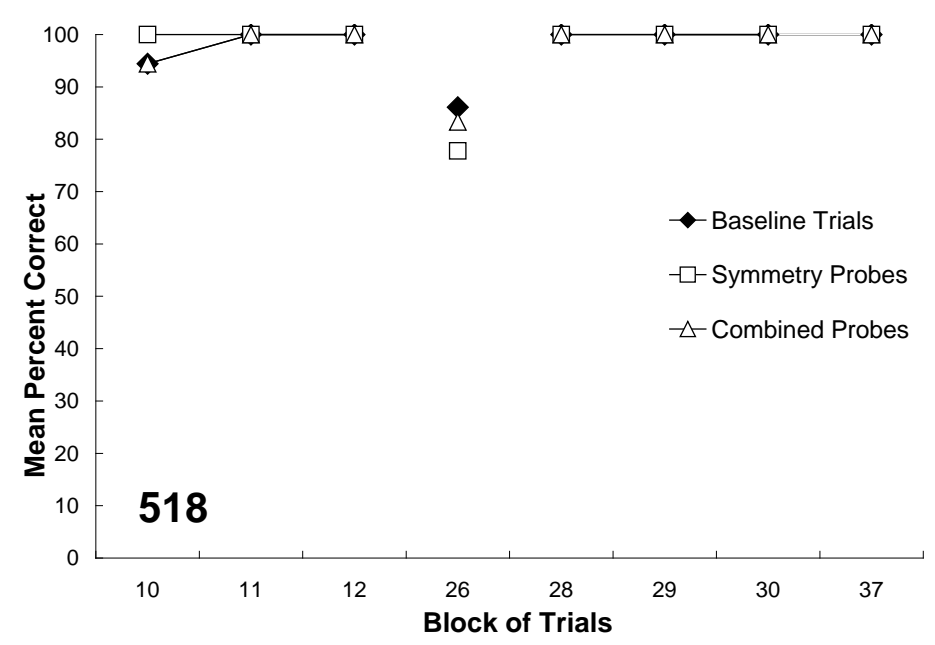

Figure 17. Mean percent correct responding for baseline and probe trials during testing for stimulus equivalence for Subject 518. Remedial training followed the $26^{\text {th }}$ block of trials. All other breaks in the data series indicate instances in which testing for stimulus equivalence alternated with testing for functional equivalence.

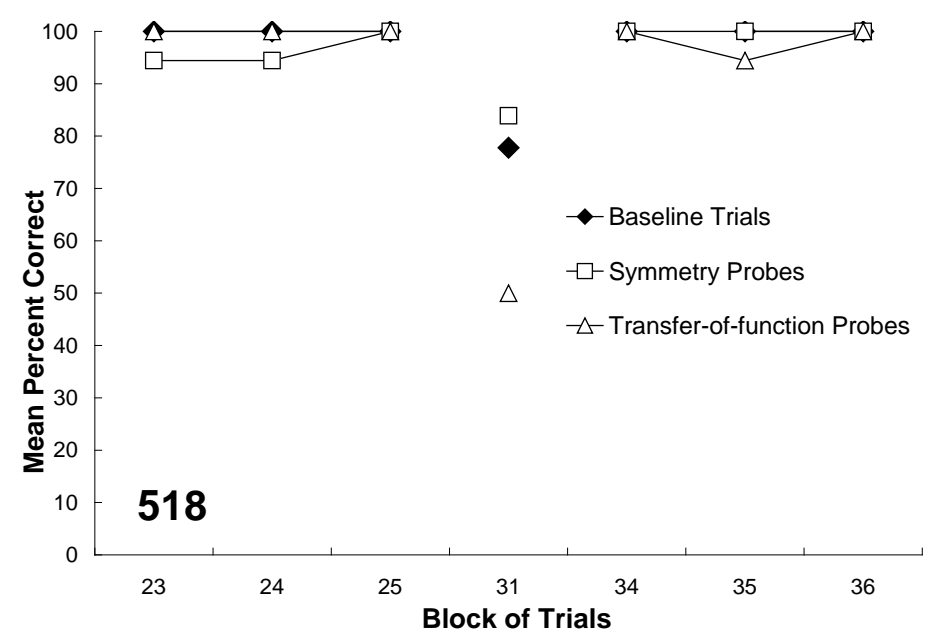

Figure 18. Mean percent correct responding for baseline and probe trials during testing for functional equivalence for Subject 518. Remedial training followed the $31^{\text {st }}$ block of trials. All other breaks in the data series indicate instances in which testing for functional equivalence alternated with testing for stimulus equivalence.

Figure 19 shows class-consistent baseline and probe responding by Subject 271 across all blocks of testing for stimulus equivalence. Figure 20 depicts her respective responding during testing for functional equivalence. 


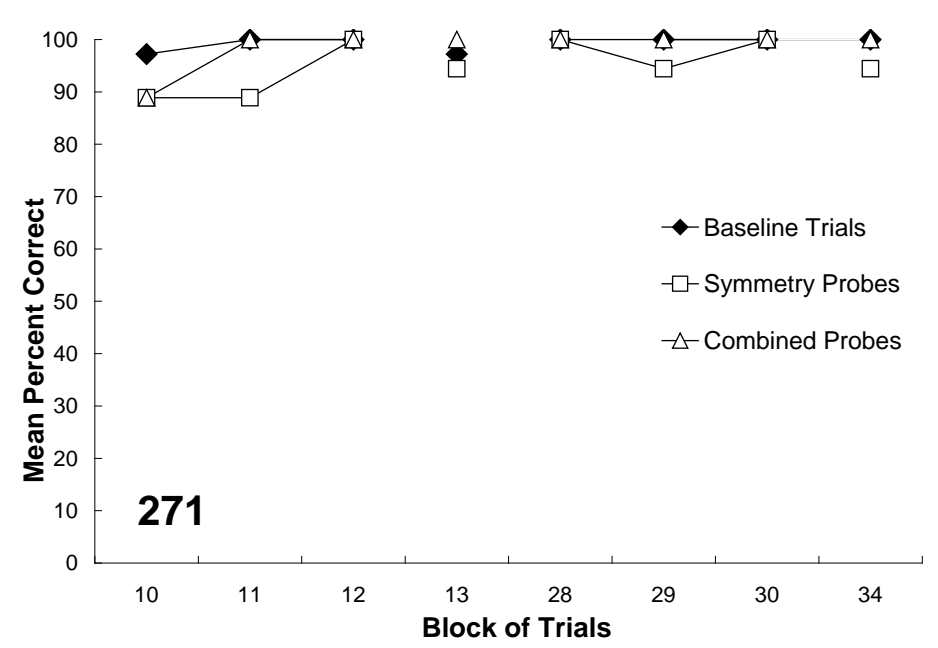

Figure 19. Mean percent correct responding for baseline and probe trials during testing for stimulus equivalence for Subject 271. Breaks in the data series indicate instances in which testing for stimulus equivalence alternated with testing for functional equivalence.

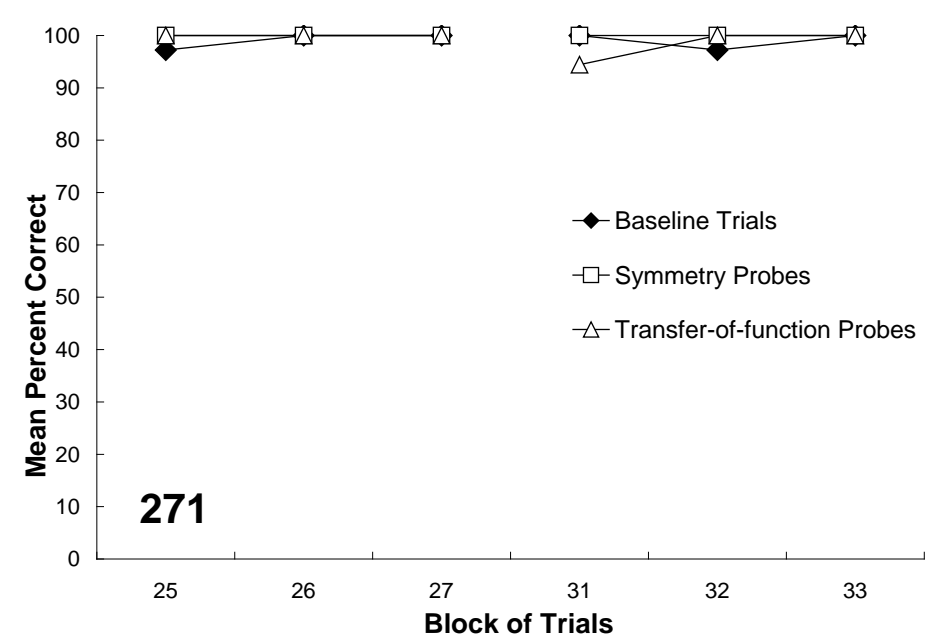

Figure 20. Mean percent correct responding for baseline and probe trials during testing for functional equivalence for Subject 271. Breaks in the data series indicate instances in which testing for functional equivalence alternated with testing for stimulus equivalence.

Figure 21 shows performance by Subject 807 across all blocks of testing for stimulus equivalence. Figure 22 shows her performance during testing for functional equivalence. The last block of testing for functional equivalence was conducted after "Gox" replaced "Tib" as a target response. Class-consistent responding was observed regardless of the change in this response. 


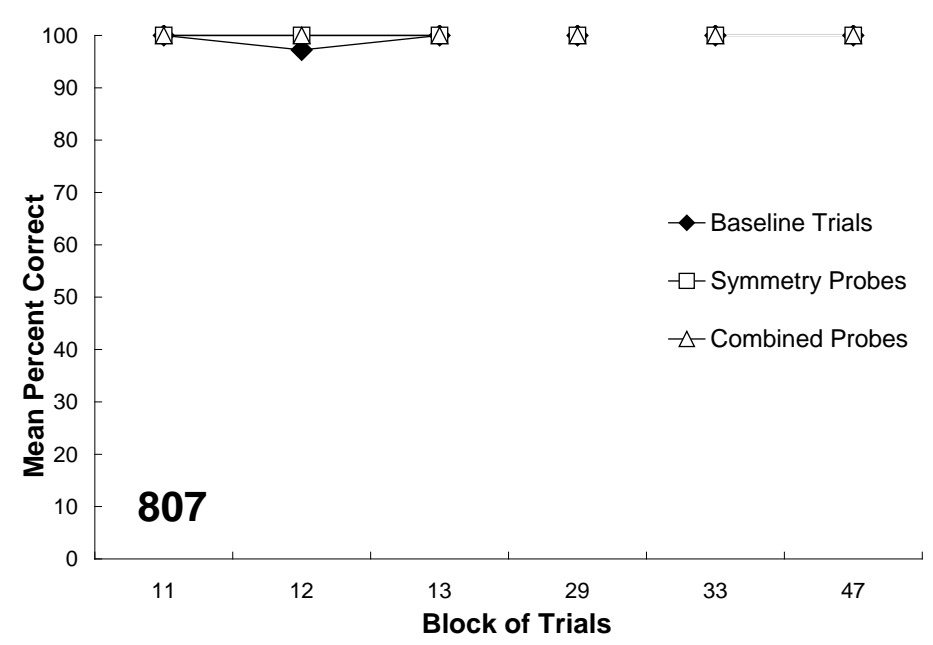

Figure 21. Mean percent correct responding for baseline and probe trials during testing for stimulus equivalence for Subject 807. Breaks in the data series indicate instances in which testing for stimulus equivalence alternated with testing for functional equivalence.

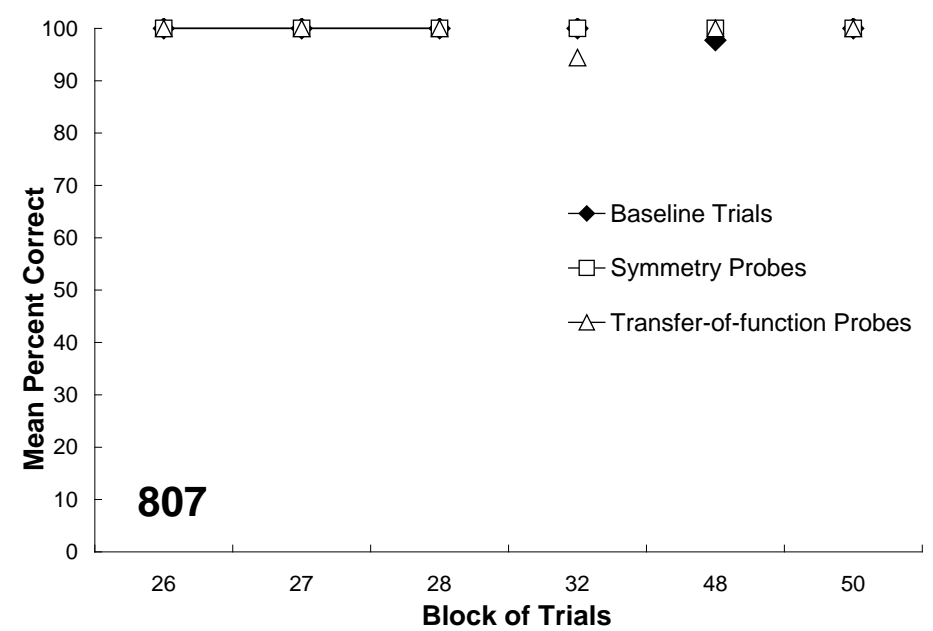

Figure 22. Mean percent correct responding for baseline and probe trials during testing for functional equivalence for Subject 807. Breaks in the data series indicate instances in which testing for functional equivalence alternated with testing for stimulus equivalence.

Baseline Training for Merging and Expansion of Classes

All subjects successfully completed baseline training for both merging and expansion of classes. Table 18 shows the number of blocks of trials completed by each subject in order to reach mastery at each stage of baseline training for either merging or expansion of classes. In the case of 
Subject 807, her last block of AE training and of FR training with absence of visual prompts were conducted after the response "Gox" replaced "Tib" as a target response.

Table 18

Blocks of Trials completed by each Subject at each stage of Baseline Training for Merging and Expansion of Classes in Experiment 2.

\begin{tabular}{|c|c|c|c|c|}
\hline Training Stage & Subject 452 & Subject 518 & Subject 271 & Subject 807 \\
\hline \multicolumn{5}{|c|}{ Merging of Classes } \\
\hline AR Training / Prompts & 5 & 2 & --- & --- \\
\hline AR Training / No Prompts & 2 & 2 & --- & --- \\
\hline AE Training & --- & --- & 3 & 4 \\
\hline \multicolumn{5}{|c|}{ Expansion of Classes } \\
\hline FR Training / Prompts & --- & --- & 7 & 3 \\
\hline FR Training / No Prompts & --- & --- & 2 & 3 \\
\hline FE Training & 3 & 2 & --- & --- \\
\hline Total Blocks of Trials & 10 & 6 & 12 & 10 \\
\hline
\end{tabular}

\section{Testing for Merging and Expansion of Classes}

Subjects 452 and 518 demonstrated merging of classes following training of simple discriminations that related independent pairs of functional and equivalence classes (AR training). Expansion of the merged classes also was successful after training of conditional discriminations linking a new stimulus to one member of each merged class (FE training).

Responding across all block of testing for merging and expansion of classes is shown in Figure 23 for Subject 452 and in Figure 24 for Subject 518. Baseline performance remained accurate 
throughout testing. Correct responding remained high for baseline trials as well as for both previously presented probes and probes for novel relations consistent with either the merged or expanded classes.

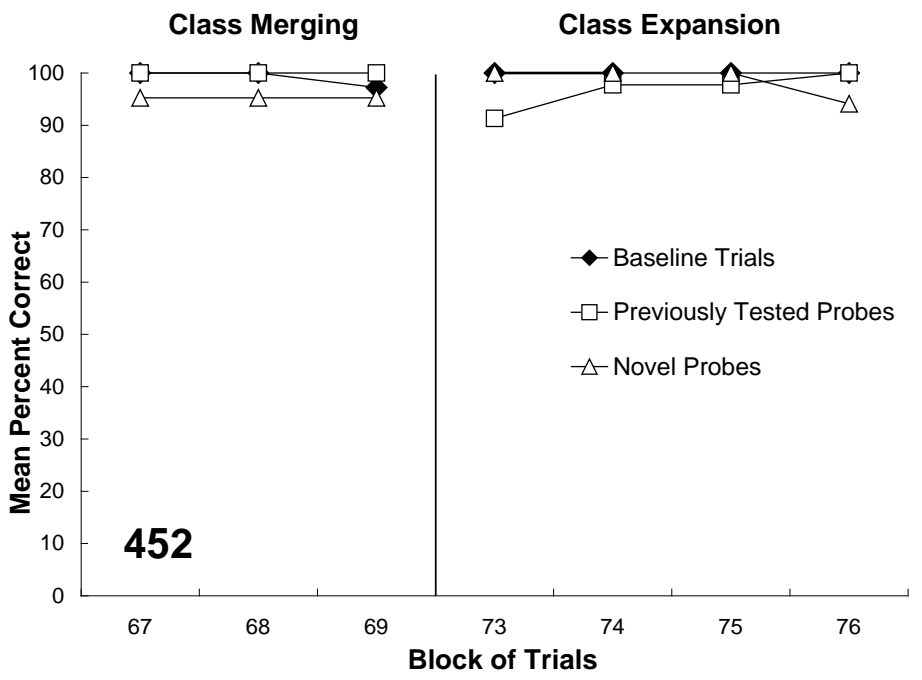

Figure 23. Mean percent correct responding for baseline and probe trials during testing for merging and expansion of classes for Subject 452.

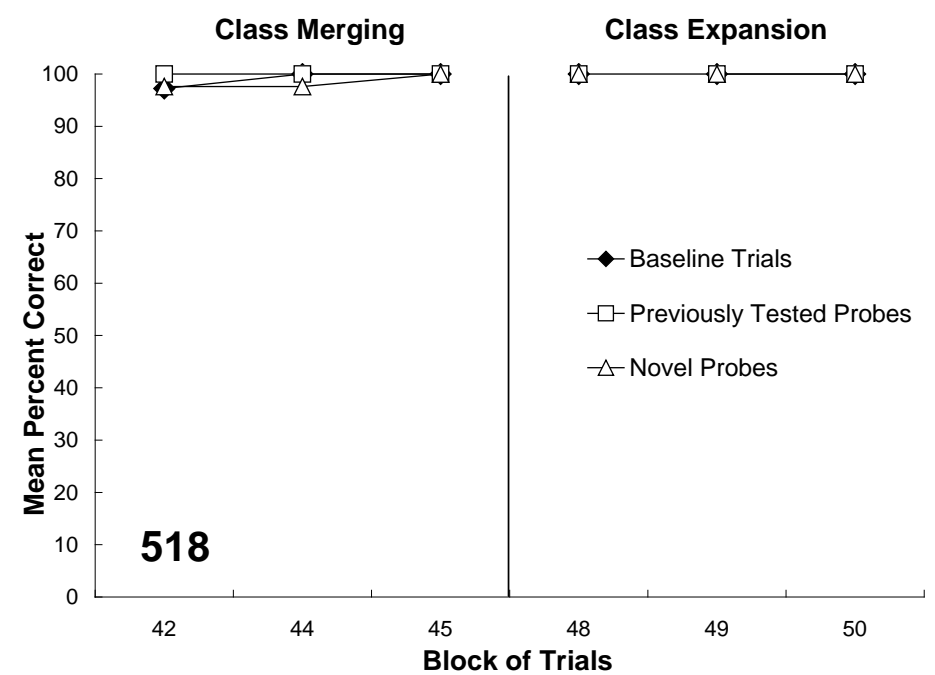

Figure 24. Mean percent correct responding for baseline and probe trials during testing for merging and expansion of classes for Subject 518. 
Subjects 271 and 807 demonstrated merging of classes following training of conditional discriminations between stimuli from independent pairs of functional and equivalence classes (AE training). The merged classes were expanded successfully after training of simple discriminations relating a new stimulus to the target response controlled of each merged class (FR training)

Figure 25 shows all blocks of testing for merging and expansion of classes for Subject 271. This subject showed class-consistent probe responding throughout both testing conditions. Baseline responding by this student, however, dropped slightly below $90 \%$ during testing for class expansion. Only two blocks of testing for class expansion were conducted due to time constraints by the participant.

Figure 26 depicts all blocks of testing for merging and expansion of classes for Subject 807. Performance by this participant in previously presented probes fell slightly below $90 \%$ correct responding during the last block of initial testing for class expansion, although her performance in novel probes aimed at testing explicitly for class expansion remained close to $100 \%$. Difficulties with recognition of the target response "Tib" were observed at this point. Subsequent testing for merging and expansion of classes after the target response "Gox" was introduced revealed highly accurate baseline and probe responding across both testing conditions. 


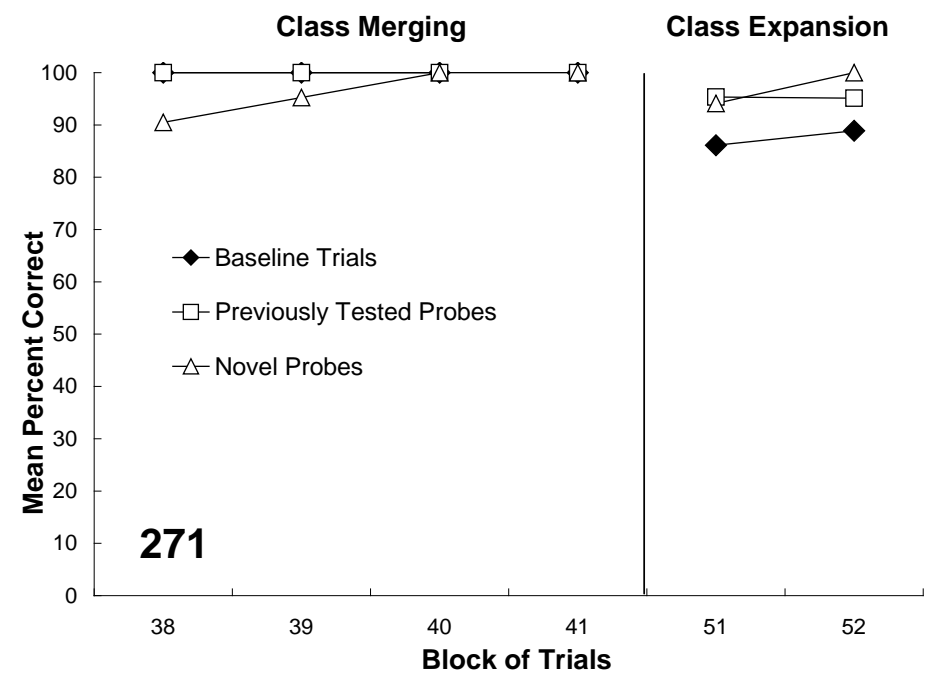

Figure 25. Mean percent correct responding for baseline and probe trials during testing for merging and expansion of classes for Subject 271.

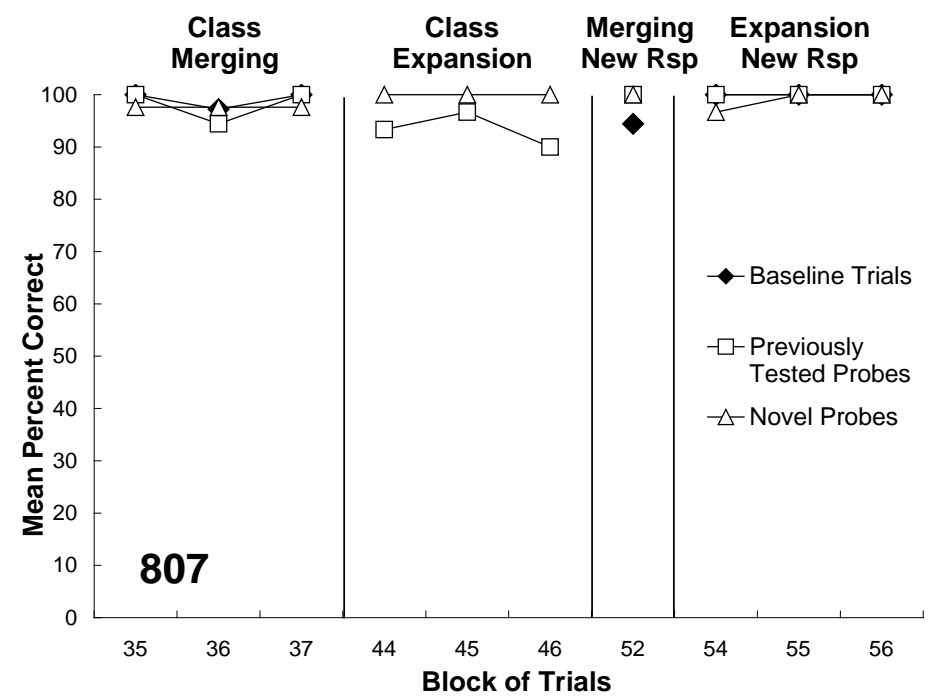

Figure 26. Mean percent correct responding for baseline and probe trials during testing for merging and expansion of classes for Subject 807.

\section{Discussion}

All subjects successfully demonstrated merged classes. Class union occurred after completion of baseline training that linked pairs of functional and equivalence classes via either a simple or a conditional discrimination. Consequently, training of responding to the two types of 
ordered pairs targeted in this experiment was effective in merging the emergent classes. The current experiment suggests that merging a functional and an equivalence class can be produced by training just one relation between both classes.

Training responses to the two types of ordered pairs examined in this experiment also was effective in expanding the merged classes by one new stimulus per class. As in the case of class merging, class expansion by training a simple or a conditional discrimination yielded positive results. Class-consistent responding for probes involving the new stimulus readily occurred following the required baseline training.

For each subject, training involving both types of ordered pairs produced highly accurate probe responding. Each type of ordered pair facilitated either merging or expansion of classes. Consequently, using simple or conditional discriminations to merge and expand classes proved effective not only across subjects but also for each individual subject.

The procedural changes introduced in this experiment successfully addressed the limitations observed in Experiment 1. All subjects mastered the baseline discriminations taught during training of functional and equivalence classes and subsequently demonstrated the targeted independent emergent classes. Subjects also maintained class-consistent responding across most alternations of testing for functional and stimulus equivalence. Remedial training effectively improved correct responding in the few instances in which baseline performance fell below mastery level during testing conditions.

Subjects progressed steadily throughout baseline training and retraining. In Experiment 1, participants completed baseline training for functional classes in 20 to 27 blocks of trials; whereas participants in Experiment 2 required 9 to 17 blocks of training. Retraining for functional classes lasted a minimum of 9 blocks of trials in Experiment 1; whereas such retraining required a maximum of 5 blocks of trials in the current experiment. As a result of successfully completing the 
functional and stimulus equivalence training, all participants were provided with the necessary conditions to proceed with merging and expansion of classes. That this merging and expansion also was successful suggests the importance of functional and stimulus equivalence training as conditions that lead to merging and expansion.

\section{General Discussion}

The current dissertation investigated conditions necessary to produce merging of functional and equivalence classes by manipulating the type of ordered pairs arranged to link elements of previously independent classes. As in previous research (e.g., Sidman et al., 1985), class union occurred reliably after training of responding to ordered pairs consisting of conditional discriminations between a stimulus from each one of the targeted classes.

Current findings expanded results by previous studies in that independent classes were related as well via training of simple discriminations. It was possible to consider this different type of ordered pair because functional classes were a focus of this study. Consequently, subjects successfully demonstrated merged classes after being taught discrimination in which an antecedent stimulus from an equivalence class served as a discriminative stimulus for a response controlled by a functional class.

\section{Relation between Functional and Stimulus Equivalence}

The current experiments aimed to provide empirical verification to suggestions by Sidman $(1990,1994,2000)$ on the formation of equivalence classes that would bring into further consideration the relation between functional and stimulus equivalence (Dube, McDonald, \& McIlvane, 1991; Sidman et al., 1989; Vaughan, 1988; Wirth \& Chase, 2002).

Sidman (2000) observed that the contingencies of reinforcement arranged during training for stimulus equivalence directly produce equivalence relations consisting of all possible ordered pairs among differential responses and stimuli that participated in baseline contingencies of 
reinforcement. This study provides empirical support for Sidman's position and expands his theoretical observations to the context of functional classes. It appears that responses also can be part of the ordered pairs trained to produce emergent relations.

Furthermore, as results from merging of functional and equivalence classes reveal, members of equivalence classes can show emergent discriminate control over formerly unrelated differential responding. In addition, stimuli from functional classes can participate in relations of combined symmetry and transitivity with members of previously independent equivalence classes. Thus, current research suggests that simple and conditional discriminations are included in the ordered pairs describing all possible emergent relations from class-specific elements of baseline contingencies of reinforcement (cf., Sidman, 2000).

These findings corroborate previous research in which stimulus equivalence was demonstrated among members of established emergent functional classes, and transfer of control of differential responding was shown by members of established equivalence classes (see Dube et al., 1991; Sidman et al., 1989; Vaughan, 1988).

This experiment also addresses the limitations of previous research on merging of functional and equivalence classes conducted by Chase et al. (2008). First, the current study directly manipulated merging of classes by relating independent classes via either simple or conditional discriminations. In addition, the type of ordered pair used for class merging was counterbalanced during class expansion across subjects. Abstract stimuli and nonsense vocal responses were used throughout current research as well in an effort to control for pre-experimental historical effects. These procedural features allowed for a more precise examination of the role of specific simple and conditional discriminations in producing class merging and expansion. 


\section{Limitations of the Current Study}

This study showed that merging of functional and equivalence classes can be produced once stable class-consistent performance is observed for independent baseline classes. Merged classes resulted from training either one simple or one conditional discrimination linking the targeted classes. In this regard, acquisition of a single ordered pair that includes elements from both classes seems sufficient to produce correct responding for all derived emergent relations. The current studies, however, did not separate the effects of a number of related variables as originally proposed (see the staggered introduction of merging of classes in Figure 2).

All available emergent classes were targeted for merging at the same time as the ordered pairs relating each functional class to an equivalence class were intermixed within the same block of training trials. Consequently, training for class merging encompassed not only the ordered pairs connecting the independent classes, but also simultaneous discriminations differentiating the ordered pairs from each other (c.f., K. J. Saunders, R. R. Saunders, Williams, \& Spradlin, 1993).

Thus, the conducted experiments did not examine whether training of the simultaneous discriminations is necessary to produce class merging (R. R. Saunders \& Green, 1999). Stimuli used to link classes, however, were trained in simultaneous and successive simple discriminations during baseline training for functional and equivalence classes. Additional research may arrange class union in a staggered fashion, as was originally planned (see Figure 2). Targeted ordered pairs would be trained one at a time, with training for each ordered pair followed by testing for class merging.

Merged classes also were expanded concurrently. The simple or conditional discriminations used to expand each merged class were trained in the same block of trials. Just as noted for merging of classes, it remains to be determined whether training of the simultaneous discriminations differentiating the ordered pairs arranged for class expansion is necessary for demonstration of subsequent emergent relations. 
Class expansion could be arranged in a staggered fashion as described above. In this case, discriminations featuring new stimuli would be trained one at a time. In contrast to procedures arranging for staggered merging of classes, it should be noted that the novel stimuli added during class expansion have not been part of any previously trained simultaneous or successive simple discrimination. This arrangement would present subjects with a more complex experimental arrangement.

Current research also conducted training for class expansion following successful merging of independent classes. A more comprehensive examination of the types of ordered pairs that produce class expansion would arrange for training of a variety of simple and conditional discriminations linking new stimuli to emergent classes before any further manipulations. Such research, however, was beyond the scope of this dissertation.

\section{Directions for Future Research}

The conducted research arranged class merging and expansion via training of simple and conditional discriminations. These discriminations included stimuli from the functional and equivalence classes, and in the case of the simple discriminations, the responses controlled by the functional classes. All class-specific stimuli were presented as antecedents during training of the baseline discriminations producing the independent, merged, or expanded classes.

As proposed in Experiment 1, if class-specific consequences are part of training for baseline discriminations, they too can be tested for membership in emergent classes. Sidman (2000) indicated that the addition of class-specific consequences arranges for these stimuli to become members of emergent classes that include the consequences when featured as discriminative or conditional stimuli (Dube \& McIlvane, 1995; Dube et al., 1987; Dube, McIlvane, Maguire, Mackay, \& Stoddard, 1989; McIlvane et al., 1992). Although the current experiments originally were designed to 
investigate this possibility, procedural problems encountered in Experiment 1 prohibited answering this question.

Future studies, therefore, should include class-specific consequences during training of either functional or equivalence classes, or such consequences can be added during class expansion conducted before or after merging the independent classes. In an examination of types of ordered pairs that modify emergent classes, it would be of interest to produce merging or expansion of classes by training discriminations that relate a class-specific consequence introduced during baseline training for one class to a stimulus or response controlled by another class.

As mentioned when discussing the results of Experiment 1, the inclusion of class-specific consequences would increase the complexity and number of probes for emergent relations presented to subjects. In this regard, it is suggested that targeted classes include relatively few members and that class-specific consequences be introduced via training of new discriminations after emergent classes are demonstrated. Thus, the number of subsequent probes to be presented will increase only after subjects have been exposed thoroughly to the training and testing procedures particular to this area of research.

Sidman (2000) suggested that responses that are differentially controlled by stimulus classes also could be presented as stimuli during probes for emergent relations. For example, the spoken words that were taught as responses in the current experiments could be played through headphones as auditory stimuli for the subjects. These stimuli would be expected to become members of subsequent emergent classes, just as in the case of class-specific antecedent and consequent stimuli presented during baseline contingencies of reinforcement. Research to this effect would more thoroughly assess Sidman's assertion that emergent relations consist of all ordered pairs that contain all positive elements that participate in a contingency of reinforcement. 
Future studies could follow the procedures described in this dissertation to produce emergent classes controlling differential responding. Tests for emergent relations can present responses as conditional or discriminative stimuli by including auditory stimuli that correspond to each target response (i.e., spoken words). The auditory stimuli could correspond to a reproduction of the subjects' utterances of the responses during the experiment. In an alternative procedure, written syllables corresponding to the target responses could serve as antecedent stimuli during probes for emergent relations.

\section{Final Remarks}

This dissertation presented a series of procedures for successfully producing independent functional and equivalence classes. Subjects maintained class-consistent performance across time, as revealed by repeated testing for functional and stimulus equivalence. In addition, the current study demonstrated that acquisition of one simple or conditional discrimination is sufficient to merge an equivalence class to a functional class, and that training of one additional simple or conditional discrimination also is sufficient to expand a merged class by adding one novel stimulus to the targeted class.

Current research contributed to our understanding of complex stimulus control in humans by exploring basic processes related to the formation, merging, and expansion of arbitrary stimulus classes. This dissertation explored generative aspects of behavior and provided support to accounts of emergent responding in terms of explicit contingencies of reinforcement. A history of reinforcement featuring specific simple and conditional discriminations was shown to effectively produce responding consistent with derived stimulus relations that were not taught explicitly.

The experiments presented here provide a model for investigating instances of adaptive human behavior that occur when novel environmental events are introduced. These experiments 
studied instances of abstract visual and vocal responding in an effort to explore conditions that may underlie language processes. 


\section{References}

Carlin, L. A., Wirth, O., \& Chase, P. N. (1998). Effects of sample-response requirements on matching-to-sample performance with humans. Experimental Analysis of Human Behavior Bulletin, 16, 2-5.

Chase, P. N. (2003). Behavioral education: Pragmatic answers to questions about novelty and efficiency. In K. A. Lattal \& P. N. Chase (Eds.), Behavior theory and philosophy (pp. 347-367). New York: Kluwer Academic/Plenum.

Chase, P. N., Ellenwood, D. W., \& Madden, G. (2008). A behavior analytic analogue of learning to use synonyms, syntax, and parts of speech. The Analysis of Verbal Behavior, 24, 31-54.

Chomsky, N. (1959). Verbal behavior. By B. F. Skinner. Language, 35, 26-58.

Dougher, M. J., \& Markham, M. R. (1994). Stimulus equivalence, functional equivalence, and the transfer of function. In S. C. Hayes, L. J. Hayes, M. Sato, \& K. Ono (Eds.), Behavior analysis of language and cognition (pp. 71-90). Reno, NV: Context.

Dougher, M. J., \& Markham, M. R. (1996). Stimulus classes and the untrained acquisition of stimulus functions. In T. R. Zentall \& P. M. Smeets (Eds.), Stimulus class formation in humans and animals (pp 137-152). Amsterdam, The Netherlands: Elsevier.

Dube, W. V., McDonald, S. J., \& McIlvane, W. J. (1991). A note on the relationship between equivalence classes and functional stimulus classes. Experimental Analysis of Human Behavior Bulletin, 9, 7-11.

Dube, W. V., \& Mcllvane, W. J. (1995). Stimulus-reinforcer relations and emergent matching to sample. The Psychological Record, 45, 591-612.

Dube, W. V., Mcllvane, W. J., Mackay, H. A., \& Stoddard, L. T. (1987). Stimulus class membership established via stimulus-reinforcer relations. Journal of the Experimental Analysis of Behavior, 47, 159-175. 
Dube, W. V., Mcllvane, W. J., Maguire, R. W., Mackay, H. A., \& Stoddard, L. T. (1989). Stimulus class formation and stimulus-reinforcer relations. Journal of the Experimental Analysis of Behavior, $51,65-76$.

Goldiamond, I. (1962). Perception. In A. J. Bachrach (Ed.), Experimental foundations of clinical psychology (pp. 280-340). New York: Basic Books.

Goldiamond, I. (1966). Perception, language, and conceptualization rules. In B. Kleinmuntz (Ed.), Problem solving (pp. 183-224). New York: Wiley.

Green, G., \& Saunders, R. R. (1998). Stimulus equivalence. In K. A. Lattal \& M. Perone (Eds.), Handbook of research methods in human operant behavior (pp. 229-262). New York: Plenum.

Halmos, P. R. (1960). Naïve set theory. Princeton, NJ: D. Van Nostrand.

Hayes, S. C., Barnes-Holmes, D., \& Roche, B. (2001). Relational frame theory: A post-Skinnerian account of buman language and cognition. New York: Kluwer Academic/Plenum.

Hrbacek, K., \& Jech, T. (1999). Introduction to set theory (3rd. ed.). New York: Marcel Dekker.

Johnson, C., \& Sidman, M. (1993). Conditional discrimination and equivalence relations: Control by negative stimuli. Journal of the Experimental Analysis of Behavior, 59, 333-347.

Keller, F. S., \& Schoenfeld, W. N. (1950). Principles of psychology. New York: Appleton-Century-Crofts.

Lazar, R. (1977). Extending sequence-class membership with matching to sample. Journal of the Experimental Analysis of Behavior, 27, 381-392.

McIlvane, W. J., Dube, W. V., Kledaras, J. B., de Rose, J. C., \& Stoddard, L. T. (1992). Stimulusreinforcer relations and conditional discriminations. In S. C. Hayes \& L. J. Hayes (Eds.), Understanding verbal relations (pp. 43-67). Reno, NV: Context.

Michael, J. (1985). Two kinds of verbal behavior plus a possible third. The Analysis of Verbal Behavior, 3, 2-5. 
Pilgrim, C., \& Galizio, M. (1996). Stimulus equivalence: A class of correlations, or a correlation of classes? In T. R. Zentall \& P. M. Smeets (Eds.), Stimulus class formation in humans and animals (pp 173-195). Amsterdam, Netherlands: Elsevier.

Saunders, K. J., Saunders, R. R., Williams, D. C., \& Spradlin, J. E. (1993) An interaction of instructions and training design on stimulus class formation. Extending the analysis of equivalence. The Psychological Record, 43, 725-744.

Saunders, R. R., Drake, K. M., \& Spradlin, J. E. (1999). Equivalence class establishment, expansion, and modification in preschool children. Journal of the Experimental Analysis of Behavior, 71, 195214.

Saunders, R. R., \& Green, G. (1999). A discrimination analysis of training structure effects on stimulus equivalence outcomes. Journal of the Experimental Analysis of Behavior, 72, 117-137.

Shahan, T. A., \& Chase, P. N. (2002). Novelty, stimulus control, and operant variability. The Behavior Analyst, 25, 175-190.

Sidman, M. (1986). Functional analysis of emergent verbal classes. In T. Thompson \& M. D. Zeiler (Eds.), Analysis and integration of behavioral unit. (pp. 213-245). Hillsdale, NJ: Lawrence Earlbaum.

Sidman, M. (1990). Equivalence relations: Where do they come from? In D. E. Blackman \& H. Lejeune (Eds.), Behaviour analysis in theory and practice: Contributions and controversies (pp. 93-114). Hillsdale, NJ: Lawrence Earlbaum

Sidman, M. (1994). Equivalence relations and behavior: A research story. Boston: Authors Cooperative.

Sidman, M. (2000). Equivalence relations and the reinforcement contingency. Journal of the Experimental Analysis of Behavior, 74, 127-146. 
Sidman, M., Kirk, B., \& Willson-Morris, M. (1985). Six-member stimulus classes generated by conditional-discrimination procedures. Journal of the Experimental Analysis of Behavior, 43, 2142.

Sidman, M., Rauzin, R., Lazar, R., Cunningham, S., Tailby, W., \& Carrigan, P. (1982). A search for symmetry in the conditional discriminations of rhesus monkeys, baboons, and children. Journal of the Experimental Analysis of Behavior, 37, 23-44.

Sidman, M., \& Tailby, W. (1982). Conditional discriminations vs. matching-to-sample: An expansion of the testing paradigm. Journal of the Experimental Analysis of Behavior, 37, 5-22.

Sidman, M., Wynne, C. K., Maguire, R. W., \& Barnes, T. (1989). Functional classes and equivalence relations. Journal of the Experimental Analysis of Behavior, 52, 261-274.

Stikeleather, G., \& Sidman, M. (1990). An instance of spurious equivalence relations. The Analysis of Verbal Behavior, 8, 1-11.

Touchette, P. E. (1971). Transfer of stimulus control: Measuring the moment of transfer. Journal of the Experimental Analysis of Behavior, 15, 347-354.

Vaughan, W., Jr. (1988). Formation of equivalence sets in pigeons. Journal of Experimental Psychology: Animal Behavior Processes, 14, 36-42.

Wirth, O., \& Chase, P. N. (2002). Stability of functional equivalence and stimulus equivalence: Effects of baseline reversals. Journal of the Experimental Analysis of Behavior, 77, 29-47.

Wirth, O., \& Chase, P. N., \& Munson, K. J. (2000). Experimental analysis of human vocal behavior: Applications of speech-recognition technology. Journal of the Experimental Analysis of Behavior, 74, 363-375. 


\section{Appendix A}

\section{Recruitment Form}

Here is an opportunity to earn extra credit by participating in a study in the Department of Psychology. We want to recruit female students who are enrolled in Psychology courses to participate in a study examining relations among visual stimuli and vocal responses. Participation will require coming to approximately 10 daily sessions. Each session is one hour to one hour and 30 minutes long. Extra credit slips valid in this course are offered in this study, and you will earn money paid as gift certificates for local businesses based on your performance in the study and your attendance to the sessions. For more information, contact Harold Lobo at hlobo@mix.wvu.edu. If you want to participate, sign up below and we will contact you and arrange the times for conducting the study. Thank you!

Name

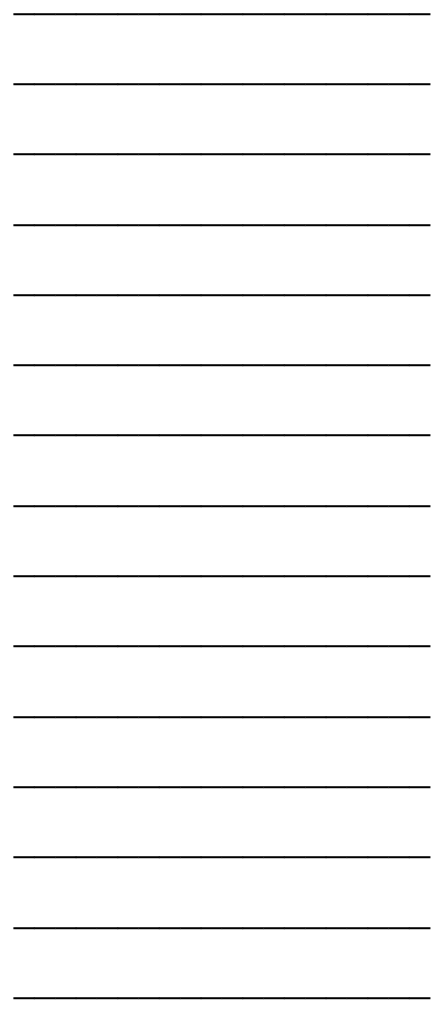

Phone

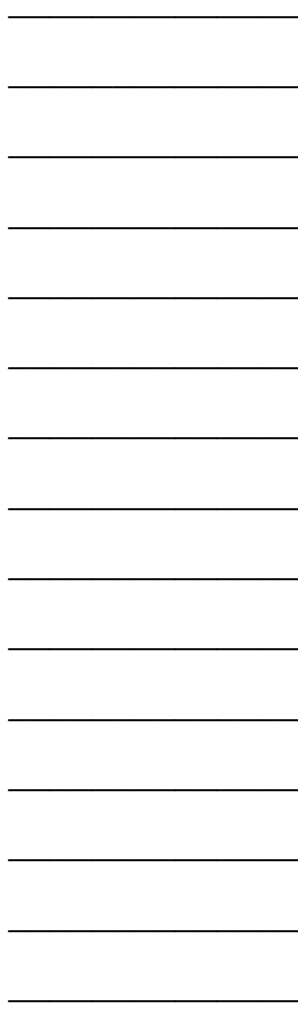

E-mail address

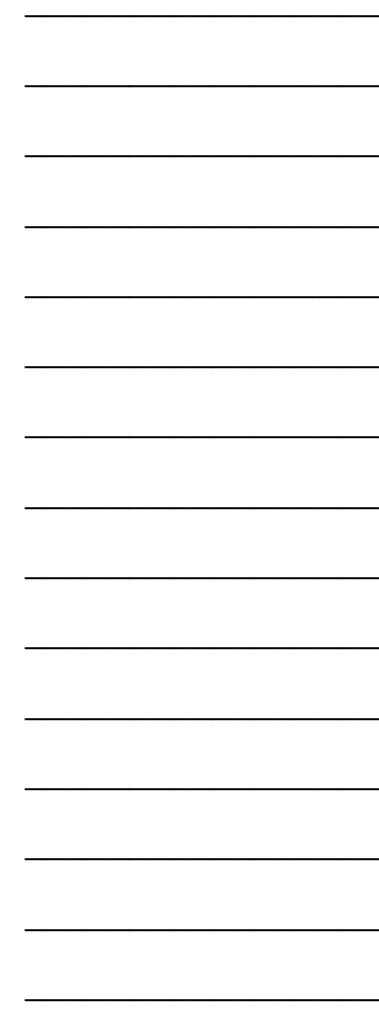

Best time to call

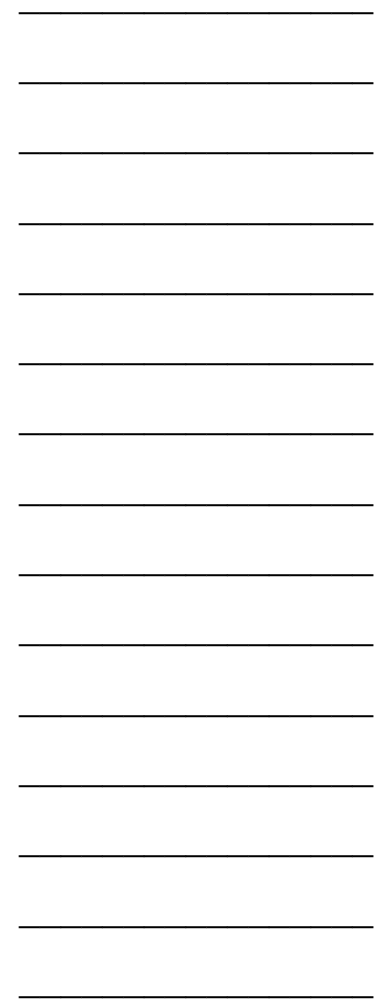


Appendix B

Consent and Information Form

\section{Relations among Visual Stimuli and Vocal Responses}

\section{$\underline{\text { Introduction }}$}

I, __ have been invited to participate in this research study, which has been explained to me by Harold Lobo or by . This research is being conducted to fulfill the requirements of a Doctoral Dissertation in Psychology at West Virginia University.

\section{Purpose of the study}

The purpose of this study is to understand more about how relations among visual stimuli and vocal responses are learned. I understand that the information collected from my participation in this study might be used in Harold Lobo's Doctoral Dissertation.

\section{Description of procedures}

This study involves playing several games on a computer. It also entails accumulating earnings that will be exchanged for gift certificates at the end of the study. I understand that the value of the gift certificates will depend on my performance on those games and my attendance to the study. I understand that, if I complete the experiment as determined by the experimenter or one of his assistants, I will also receive extra-credit slips valid in Psychology courses according to each course's policy.

I have been informed that this study will take approximately fifteen hours for me to complete. I understand that I will participate in approximately one session per day.

I understand that because of the experimental protocol, it is important for me to come every day at my agreed upon time. I understand that if I miss a session, I will be asked to come in for a make-up session within a week of the missed session. I also understand that if I miss two or more sessions, or if I do not call in advance of missing a session, I may be dropped from the experiment. If I become ineligible to continue because of missed sessions, I understand that I will neither receive the extra-credit slips nor earnings towards the gift certificates based on my attendance to the study.

I understand that I will be compensated for my participation in the study at the end of the experiment, and that the experimenter or his assistants will keep careful track of my earnings and attendance throughout the experiment. Approximately 8 students are expected to participate in this study.

Version Date:

Page 1 of 3

Initials 


\section{Relations among Visual Stimuli and Vocal Responses}

\section{$\underline{\text { Risks and discomforts }}$}

There are no known or expected risks from participating in this study, except for the mild frustration and/or boredom that I might experience in connection with my performance on the computer games.

\section{$\underline{\text { Alternatives }}$}

I understand that I do not have to participate in this study and that I will not suffer any type of negative consequences if I decline to participate in the study.

\section{$\underline{\text { Benefits }}$}

I understand that this study is not expected to be of direct benefit to me, but that the knowledge obtained in this study may be of benefit to others. I will receive gift certificates based on my performance and attendance in the study, and extra-credit slips upon completion of the experiment, as determined by the experimenter or one of his assistants.

\section{Financial considerations}

In this study, I will earn $\$ 0.01$ (one cent) for each correct response on the computer games. I will receive gift certificates for the amount of money that I earn based on my performance after I finish my participation in the study.

I understand that, if I complete the experiment, as determined by the experimenter or one of his assistants, I will receive a $\$ 3.00$ bonus for every session I attend.

I understand that I may be able to earn approximately $\$ 7$ in gift certificates per session of participation, and that this amount includes both my performance earnings and my attendance bonus.

\section{Contact persons}

For more information about this research, I can contact Harold Lobo at hlobo@mix.wvu.edu, or his supervisor, Dr. Philip N. Chase at (304) 293-2001, ext. 31626. For information regarding my rights as a research subject, I may contact the Executive Secretary of the Institutional Review Board at (304) 2937073.

\section{Confidentiality}

I understand that any information about me obtained as a result of my participation in this research will be kept as confidential as legally possible. In any publications that result from this research, neither my name nor any information from which I might be identified will be published without my consent.

Version Date:

Page 2 of 3

Initials 


\section{Relations among Visual Stimuli and Vocal Responses}

\section{Voluntary participation}

Participation in this study is voluntary. I understand that I am free to withdraw my consent to participate in this study at any time and that such refusal to participate will not affect my student status at West Virginia University or my class standing or grades. Refusal to participate or withdrawal will not involve any penalty to me. I have been given the opportunity to ask questions about the research, and I have received answers concerning areas I did not understand. In the event that new information becomes available that may affect my willingness to continue to participate in the study, this information will be given to me so that I may make an informed decision about my participation.

Upon signing this form, I will receive a copy.

I willingly consent to participate in this research.

Signature of Subject or Subject's Legal Representative

Signature of Investigator or Investigator's Representative
Date

Time

Time

Version Date:

Page 3 of 3
Initials 


\section{Appendix C}

Welcome Message

\section{Welcome to the Human Behavior Laboratory!}

As part of this study, you will be asked to play a series of games on the computer that will earn you gift certificates for local businesses. You will earn one cent towards a gift certificate every time that you make a correct response. Gift certificates will be given to you at the end of the experiment.

Please get comfortable before the session begins. When you are ready to start this series of games, click the button below. When this series is over, the computer will prompt you to call the experimenter by knocking on the small wooden door located to your right. 


\section{Appendix D \\ Description of Task for Selecting Preferred Consequences}

Some logos for local businesses will be shown next. Please choose the top four logos for which you would like to receive gift certificates.

The logos you choose will be used during the rest of the experiment to represent your earnings. Specifically, correct responses in the computer games for this experiment will be followed by the logos you select.

Each correct response will add one cent to the value of the respective gift certificate. When you are ready to choose your preferred local businesses, please click the button below. 
Appendix E

Description of the Reduction in the Presentation of Differential Consequences

Today the messages and the tones telling you that you responded correctly or incorrectly will appear only occasionally. Most of the times, an empty screen will appear after a trial has ended.

You will not be able to know how much you earned during this block of games, but the computer will keep track of your earnings for you. As before, you will earn one cent every time that you make a correct response.

Remember that your earnings will be added to your selected gift certificates. 


\section{Appendix F}

Instructions for the MTS Demonstration Trials in Experiment 1

\section{Demonstration of a Correct Sequence}

As you saw in the previous demonstration, you will have to wait up to $\mathbf{3}$ seconds for the first figure to appear on the screen. As soon as the first figure appears, you can click on it to make the three additional figures visible. If you click anywhere on the screen before the first figure appears, you will have to wait longer.

If you click the correct figure from among the three additional figures, a business logo will appear along with a distinctive tone. Only one figure is correct on each trial. The logo and the tone will disappear automatically. There is nothing you can do to make them disappear. Every time that you make one correct response, one cent will be added to the gift certificate that corresponds with the displayed logo. The total amount of your earnings will be displayed at the end of each set of games.

\section{Demonstration of an Incorrect Sequence}

As you saw again, you have to wait up to $\mathbf{3}$ seconds for the first figure to appear on the screen. As soon as the first figure appears, you can click on it to make the three additional figures visible. Remember that if you do not wait long enough and you click anywhere before the first figure appears, you will have to wait longer.

If you click an incorrect figure from among the three additional figures, the symbol “ $x$ ” will appear along with a distinctive tone. No money towards your gift certificates can be earned by selecting incorrect figures. Remember that only one figure is correct on each trail. The " $\boldsymbol{x}$ " and the tone will disappear automatically. Nothing you can do will make them disappear. 


\section{Appendix $G$}

Instructions for the MTS Demonstration Trials in Experiment 2

\section{Demonstration of a Correct Sequence}

As you saw in the previous demonstration, you will have to wait up to $\mathbf{3}$ seconds for the first figure to appear on the screen. As soon as the first figure appears, you can click on it to make the three additional figures visible. If you click anywhere on the screen before the first figure appears, you will have to wait longer.

If you click the correct figure from among the three additional figures, the symbol " $\checkmark$ " will appear along with a distinctive tone. Only one figure is correct on each trial. The " $\checkmark$ " and the tone will disappear automatically. There is nothing you can do to make them disappear. Every time that you make one correct response, one cent will be added towards your gift certificates. The total amount of your earnings will be displayed at the end of each set of games.

\section{Demonstration of an Incorrect Sequence}

As you saw again, you will have to wait up to $\mathbf{3}$ seconds for the first figure to appear on the screen. As soon as the first figure appears, you can click on it to make the three additional figures visible. Remember that If you do not wait long enough and you click anywhere before the first figure appears, you will have to wait longer.

If you click an incorrect figure from among the three additional figures, the symbol “ $\boldsymbol{x}$ ” will appear along with a distinctive tone. No money towards your gift certificates can be earned by selecting incorrect figures. Remember that only one figure is correct on each trial. The " $\boldsymbol{x}$ " and the tone will disappear automatically. Nothing you can do will make them disappear. 